(C) 2020 by the Arizona Board of Regents on behalf of the University of Arizona. This is an Open Access article, distributed under the terms of the Creative Commons Attribution licence (http://creativecommons. org/licenses/by/4.0/), which permits unrestricted re-use, distribution, and reproduction in any medium, provided the original work is properly cited.

\title{
BOUNDING MIDDLE PERIOD CEMETERY USE IN SAN PEDRO DE ATACAMA, CHILE
}

\author{
William J Pestle ${ }^{1 *(1)} \cdot$ Christina Torres-Rouff ${ }^{2,3}$ - Gonzalo Pimentel ${ }^{4,5} \cdot$ Mark Hubbe $^{3,6}$ \\ ${ }^{1}$ University of Miami, Department of Anthropology, Coral Gables, FL, USA \\ ${ }^{2}$ University of California Merced, Department of Anthropology and Heritage Studies, Merced, CA, USA \\ ${ }^{3}$ Universidad Católica del Norte, Instituto de Arqueología y Antropología, San Pedro de Atacama, Chile \\ ${ }^{4}$ Fundación Patrimonio Desierto de Atacama, San Pedro de Atacama, Chile \\ ${ }^{5}$ Universidad de Tarapacá, Arica, Chile \\ ${ }^{6}$ The Ohio State University, Department of Anthropology, Columbus, OH, USA
}

\begin{abstract}
The San Pedro de Atacama oases, located in northern Chile's hyperarid Atacama Desert, have been occupied for at least 3000 years. Here, we examine cemetery use in the oases, with emphasis on the Middle Period (ca. AD 400-1000). By modeling of a large corpus $(n=243)$ of radiocarbon dates, over $90 \%$ of which are direct AMS assays of human bone collagen, we attempt to establish a temporal framework by which to explore the establishment of formalized social inequality in this period. Modeling of these dates at three locally defined scales (all ayllus, inter-ayllu, and intra-ayllu) permit heretofore unavailable insights into the chronological and spatial dimensions of life and mortuary activity in the oases and allow us to better contextualize patterns of social inequality during the dynamic Middle Period. The results of this modeling indicate two distinct peaks of occupation during the Middle Period in San Pedro and document significant temporal variability in cemetery use patterns on both inter- and intra-ayllu scales. These results stress the importance of local social and environmental factors to the occupation of the oases and provide crucial chronological structure for future archaeological and bioarchaeological research in the region.
\end{abstract}

KEYWORDS: Andes, cemeteries, Chile, chronology, Middle Period.

\section{INTRODUCTION AND BACKGROUND}

At the northern end of the Atacama salt flat, the San Pedro and Vilama Rivers feed a series of small oases in the otherwise hyperarid Atacama Desert of northern Chile (Figure 1). These oases have been host to some of the longest human occupations in the region, with the earliest evidence of habitation dating to the Formative Period (ca. $1200 \mathrm{BC}$ ), if not earlier (Núñez 1991; Costa Junqueira and Llagostera 1994; Llagostera 2004). The initial occupation of the oases was associated with the progressive expansion and adoption of horticultural practices by human groups from the nearby puna (Núñez et al. 2005), and the adoption of practices of arboriculture and silvopastoralism in the oases (McRostie 2014). This human presence slowly came to occupy most, if not all, of the arable land of the different San Pedro de Atacama oases and, by the beginning of the Spanish colonial period, the pattern of occupation was largely similar to that seen at present (Llagostera and Costa Junqueira 1999; Torres-Rouff and Hubbe 2013).

Permanent human presence in the region is evidenced by both habitation sites and large cemeteries. The cemeteries, in particular, have been at the center of discussions about human presence and demography, as they are thought to reflect conscious decisions about land occupancy, and are, in general, less disturbed by more recent human activity than the habitation sites. As such, there has long been a strong emphasis on the study of prehistoric Atacameño life through the study of burials and mortuary offerings from the many cemeteries in the region (e.g. Le Paige 1964; Llagostera 2004).

\footnotetext{
*Corresponding author. Email: w.pestle@miami.edu
} 


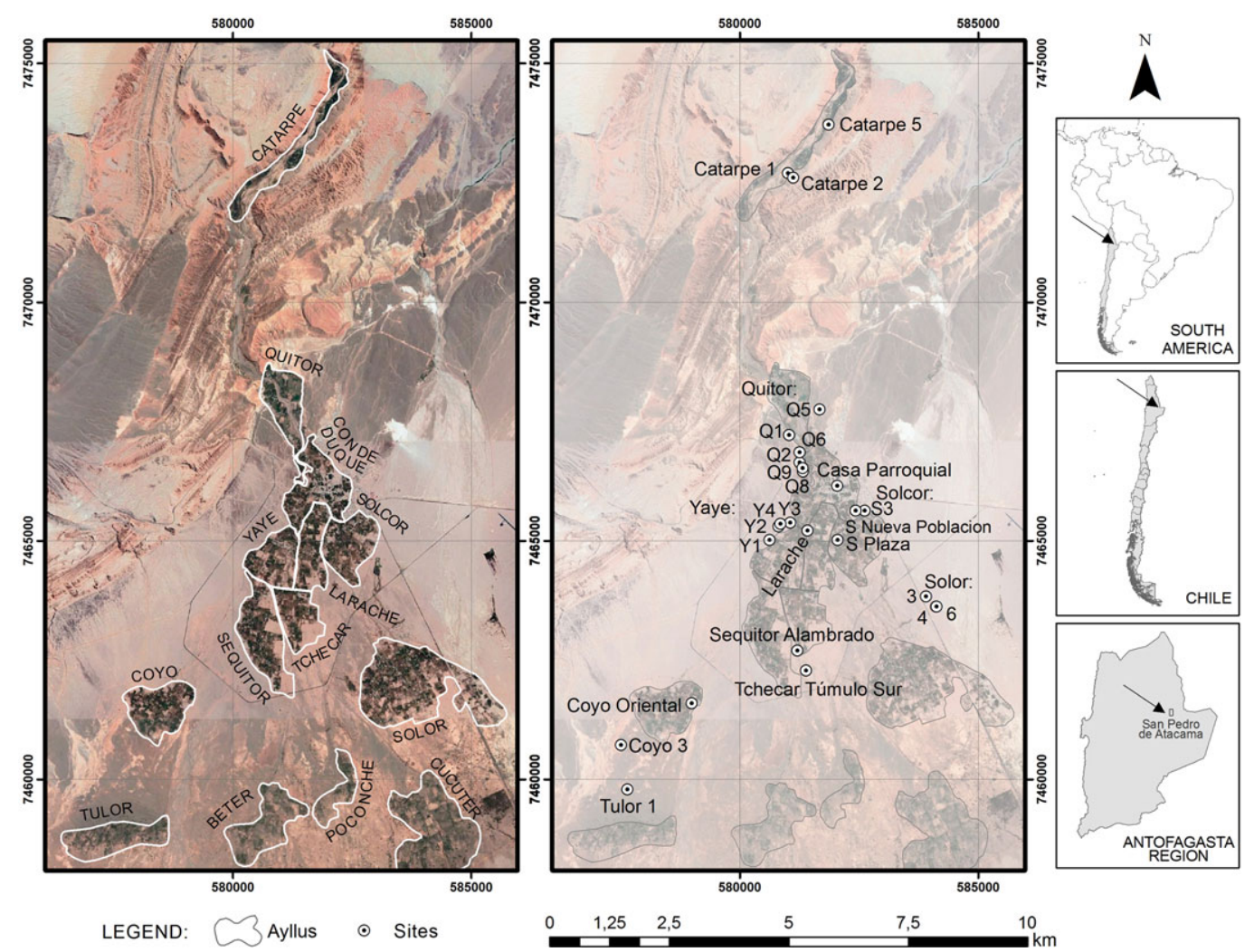

Figure 1 Map of San Pedro de Atacama, Chile, with locations of ayllus and sites discussed in text noted.

Human occupation of the oases has traditionally been divided into cultural phases based on changes in local material culture, particularly ceramic typologies and the presence of foreign or exotic objects. The deep and well-preserved archaeological evidence of human presence has led to a long history of the exploration of the chronology of the San Pedro de Atacama oases. The 1960s saw the development of work by pioneers in modern Chilean archaeology, most notably Mario Orellana $(1962,1963,1964)$ and Lautaro Núñez $(1963,1965,1966)$ as well as by Father Gustavo Le Paige (1963, 1972/1973), an amateur archaeologist and the village priest. In 1963, a number of Andean archaeologists came together for the "Congreso Internacional de Arqueología de San Pedro de Atacama" (Niemeyer 1963), now embraced as the second meeting of the Chilean Archaeological Society (Congreso Nacional de Arqueología Chilena; Campbell 2015), at which they presented research covering the entire occupation of the oases. Following this, a consensus was reached that established a local framework of phases for San Pedro de Atacama that was synchronized with both neighboring areas and the larger patterns of south-central Andean prehistory (Niemeyer 1963; Orellana 1963, 1964). These initial proposed phases were refined by Tarragó $(1968,1989)$ through ceramic seriation, and subsequently given an absolute chronological framework using a small number of radiocarbon dates (Núñez 1966, 1976; Le Paige 1976) and the thermoluminescence dating of a large sample of ceramic types (Berenguer et al. 1986, 1988). 
More recently, efforts have been made to expand the absolute chronology of San Pedro de Atacama through analysis of a suite of radiocarbon dates of human skeletal material (Hubbe et al. 2011; Torres-Rouff and Hubbe 2013), which have indicated that the limits of the local cultural phases are harder to define than previously suggested. Analysis of these radiocarbon dates for the oases' cemeteries have demonstrated that (1) multiple cemeteries have use-lives that span cultural phases, and (2) some cemeteries traditionally associated with different phases in fact exhibit concurrent occupation, calling attention to the fact that there is significant cultural diversity among, and within, individual oases in each supposed cultural phase (Torres-Rouff and Hubbe 2013).

The significance of the periodization of San Pedro's prehistory is tied not only to local developments but also to the role the oases played in the larger context of social developments in the region. Throughout its prehistory, the oases were an important node on the trade network that connected the region, through which passed goods and influence of the large polities that shaped the region's development, and from which flowed locally manufactured goods of regional importance (e.g., Núñez and Dillehay 1995; Llagostera 1996; Gallardo et al. 2017). In this context, San Pedro de Atacama's Middle Period (AD 400-1000) has been of particular scholarly interest due to the wide distribution of characteristic goods from this period of the oases throughout the region. Much like the broader Andean Middle Horizon (AD 600-1000), the Middle Period has been characterized as a time of prosperity, peace, the growth of social complexity and inequality, and burgeoning intra- and inter-regional interaction (e.g., Llagostera 2004; Núñez 2007; Isbell 2008; Castro et al. 2016). It should be noted, however, that the timing and manifestation of the Middle Period in San Pedro is not entirely consonant with the Middle Horizon of the Andes writ large.

Of late, a consensus has emerged among researchers regarding the relationship that might have existed between the Tiwanaku polity and the peoples of San Pedro de Atacama. Early research explored the notion that San Pedro operated as a colony of the large altiplano polity and significant emphasis was placed on the importance of this relationship in the oases' rise to prominence at this time (e.g., Browman 1980; Serracino 1980; Orellana 1985; Oakland 1992; Kolata 1993: 275-280; Núñez and Dillehay 1995: 98-106; Torres and Conklin 1995). However, it is now considered likely that the relationship was mediated by other individuals and via other areas, for example Cochabamba, which would have served as an indirect connection between the altiplano state and the oases (Browman 1997; Stovel 2001; Uribe et al. 2016). Importantly, this new framework shifts the emphasis away from a single power dynamic to the role of broader networks of circulation and exchange in the larger region at this time. This then, creates a system in which the peoples of San Pedro de Atacama interacted and prospered while also serving as an engine propelling the ideology and iconography of the Tiwanaku polity across the southern Andes. Moreover, the Middle Period in San Pedro shows marked differences with the Middle Period of other regions, for example, in Arica, in Chile's northernmost region, attesting to a great deal of dynamism and local and regional heterogeneity in a period broadly characterized by the same (e.g., Berenguer 1998; Uribe and Agüero 2001, 2004; Agüero 2004; Agüero and Uribe 2014; Muñoz et al. 2016; Salazar et al. 2014; Uribe et al. 2016). Ultimately, we agree with this dynamic vision of the Middle Period, a time that is not necessarily bounded by the direct presence of a Tiwanaku outpost or an abundance of Tiwanaku goods in any given locality, 
but is rather understood as being correlated with, and/or the consequence of, a complex meshing of ideological flows and economic exchange.

\section{STATEMENT OF PROBLEM}

The simplified chronology established for the local cultural phases, which can serve to equate specific sites or specific material objects to periods, has been essential in defining the broad strokes of local prehistory. However, we also suspect that it may have obscured nuances internal to the periods. As such, to be able to contribute to a larger discussion about the ways in which inequality shaped life experiences in the San Pedro de Atacama oases (e.g., Llagostera et al. 1988; Neves and Costa 1998; Torres-Rouff 2011; Hubbe et al. 2012; Figueroa et al. 2013; Salazar et al. 2014; Torres-Rouff et al. 2018), and to connect phenomena occurring in the oases with happenings in the broader Andean region, a more nuanced mapping of the chronological dimensions of San Pedro de Atacama's prehistory is required. Indeed, a better understanding of the temporal patterns of the San Pedro cemeteries, and the individuals within them, forms a foundational aspect of the study of social inequalities in the ancient southern Andes by allowing us to explore the ways in which inequality manifests in the body and the mortuary context over time and space.

Here, we aim to contribute to the discussion and identification of inequalities in the San Pedro de Atacama oases over time through the establishment of a detailed chronological framework for the main oases and principal cemeteries that represent the local archaeological record (Figure 1). Through our intensive radiocarbon dating program we aim to add nuance to the discussion and contextualize cemetery use in the different ayllus. ${ }^{1}$ This will allow us to explore the potential contemporaneity of cemeteries in each ayllu and, ideally, the potential motivations behind distinct burial grounds in shared geographic and cultural spaces. Specifically, we argue that these more detailed data allow us to explore the Middle Period as a discrete phenomenon in the oases and, further, provide a basis for archaeologists to better classify and establish the temporal dimensions of the Middle Period and subsequent phases in San Pedro de Atacama as they relate to larger patterns in the south-central Andes.

\section{MATERIALS AND METHODS}

We began our assessment of temporal patterns of Middle Period human activity in San Pedro de Atacama with a database of 243 radiocarbon dates, almost all of which were obtained from bone collagen of securely contextualized human burials, representing eleven ayllus and 27 cemeteries. Of these, 121, which are thought to be of Middle Period cultural affiliation, are reported here for the first time, and a further 96, which represent the broader sweep of occupation of the oases, were generated in the past decade by Torres-Rouff and Hubbe (2013). In both the present study and Torres-Rouff and Hubbe (2013), selection and collection of samples for dating were performed concurrently with osteological assessment of remains and the collection of samples for stable isotope analyses. Target samples of dense cortical bone were removed using sterilized diamond cutoff wheels, with samples stored in aluminum foil and sterile sample bags.

\footnotetext{
${ }^{1}$ Ayllu refers to the traditional form of Andean kin-based community structure. The ayllu reflects a political grouping and one built on lineage (ascriptive descent groups; Cock 1981; Abercrombie, 1998), but also refers to a territory, as it is always tied to a place, and importantly, these boundaries are typically associated to a huaca or sacred space tied to the ancestors of a group (Goldstein 2000: 185). In the case of San Pedro de Atacama this frequently corresponds to a naturally differentiated oasis, as is the case of the ayllus of Solor, Tulor, or Coyo in addition to internal distinctions marked by territorial features.
} 
Dating of those samples reported here for the first time, as well as the majority (67/96) of those from Torres-Rouff and Hubbe (2013), was performed at the National Science FoundationAccelerator Mass Spectrometry Laboratory at the University of Arizona. The remaining 29 samples presented in Torres-Rouff and Hubbe (2013) were dated by Beta Analytic. Finally, for the purposes of modeling, we included 26 published radiocarbon dates (20 from cemeteries and six from habitation sites) derived from other studies in the oases conducted over the past decades (Núñez 1976; Llagostera et al. 1984, 1988; Baron 1986; Costa Junqueira 1988; Torres et al. 1991; Oakland 1992; Llagostera 1995). The material/nature of the samples and the methods of sample selection and collection for these different studies varied, as did the laboratories performing the analysis; indeed, in some cases, there is very limited information available pertaining to said samples. While acknowledging these shortcomings, the inclusion of these dates allows us to obtain an understanding of the broad sweep of occupation of the ayllus, given that these include the putatively earliest and latest occupation of the region. Regardless of source, we calibrated all available dates following the procedures outlined below. Sample details are provided in Table 1. In nine instances, we possessed multiple dates for a given tomb, burial, or individual, all of which are noted as such (with an $*$ ) in the tables.

Our initial model (all ayllus) considered all available dates in an attempt to bound the Middle Period phenomenon (phase) in the oases in the broadest possible sense, but was not performed in any attempt to establish limits of settlement of the oases per se, as our sampling strategy was designed with an emphasis on presumed Middle Period sites. The sites included in this study that are considered as representative of the Middle Period were defined as such based on the archaeological presence of artifacts associated with this period (Berenguer et al. 1986; Llagostera et al. 1988; Oakland 1992; Torres-Rouff and Hubbe 2013). However, not all the individuals from the sites are directly associated with diagnostic objects from this period, and therefore it is possible that, if cemeteries spanned beyond the boundaries of the Middle Period, our study will not be able to accurately detect the length of the period in the Atacama oases. While this can be seen as a potential limitation in our study, we argue that the continuity of use of cemeteries over time is a stronger indicators of local social cohesion than the individual associations with diagnostic artifacts, especially in the context of the recent debates about the nature of the Middle Period in the oases (e.g., Berenguer 1998; Uribe and Agüero 2001, 2004; Agüero 2004; Agüero and Uribe 2014; Salazar et al. 2014; Uribe et al. 2016). As such, they represent local occupations that visibly interacted with the Middle Period phenomenon in the oases and serve as the ideal evidence of human spread during the period. Thus, the goal of the first model was to establish the temporal distribution of the occupation of the oases during what is classically seen as the Middle Period, with realization that the inclusion of some known non-Middle Period dates would tend to overestimate the boundaries of the period/phase. Prior to initiating this model, outliers (defined here as those samples with uncalibrated radiocarbon age Z-scores outside 2 standard deviations) were removed, reducing the sample size to 229 individual dates, with removed outliers noted as such in Table 1. Calibration and modeling were performed using OxCal v 4.3.2 (Bronk Ramsey 2009) and the SHCall3 curve (Hogg et al. 2013). Given the lack of evidence for marine foodstuff consumption in the oases (Pestle et al. 2016, 2017), no marine reservoir was employed in calibration. Two sigma (95.4\%) ranges are presented for both individual samples and phase boundaries in all resulting tables and figures. Model and individual sample agreement indices for this iteration are presented in Table S1. Samples with agreement indices falling below the recommended cutoff value of $60 \%$ (Bronk Ramsey 1995, 2009) were subsequently removed, and models re-run. 
Table 1 List of individuals included in present analysis, with provenience information, radiocarbon dates, and outlier designations.

\begin{tabular}{|c|c|c|c|c|c|c|c|c|c|c|c|}
\hline \multirow[b]{2}{*}{ Ayllu } & \multirow[b]{2}{*}{ Site } & \multirow[b]{2}{*}{$\begin{array}{c}\text { Sample ID } \\
\text { (site \& } \\
\text { burial number) }\end{array}$} & \multirow[b]{2}{*}{ Source } & \multirow[b]{2}{*}{ Lab \# } & \multirow[b]{2}{*}{ Material } & \multirow[b]{2}{*}{ Radiocarbon age } & \multirow[b]{2}{*}{ (sd) } & \multirow[b]{2}{*}{${ }^{13} \mathrm{C}$} & \multicolumn{3}{|c|}{ Outlier? } \\
\hline & & & & & & & & & All ayllus & Inter-ayllu & Within ayllu \\
\hline$\overline{\text { Catarpe }}$ & Catarpe 1 & 294 & $\begin{array}{c}\text { Torres-Rouff \& } \\
\text { Hubbe } 2013\end{array}$ & X14963A & Bone collagen & 752 & 43 & -12.6 & Yes & No & \\
\hline Catarpe & Catarpe 1 & 2397 & $\begin{array}{c}\text { Torres-Rouff \& } \\
\text { Hubbe } 2013\end{array}$ & X14964A & Bone collagen & 689 & 43 & -13.8 & Yes & No & \\
\hline Catarpe & Catarpe 1 & 764 & $\begin{array}{c}\text { Torres-Rouff \& } \\
\text { Hubbe } 2013\end{array}$ & Beta-293923 & Bone collagen & 620 & 30 & -12.7 & Yes & No & \\
\hline Catarpe & Catarpe 2 & 1753 & $\begin{array}{c}\text { Torres-Rouff \& } \\
\text { Hubbe } 2013\end{array}$ & Beta-251747 & Bone collagen & 1220 & 40 & -13.8 & No & No & \\
\hline Catarpe & Catarpe 2 & 1801 & $\begin{array}{c}\text { Torres-Rouff \& } \\
\text { Hubbe } 2013\end{array}$ & Beta- 251748 & Bone collagen & 1030 & 40 & -12.3 & No & No & \\
\hline Catarpe & Catarpe 2 & 1850 & $\begin{array}{c}\text { Torres-Rouff \& } \\
\text { Hubbe } 2013\end{array}$ & Beta-251749 & Bone collagen & 770 & 40 & -12.5 & Yes & No & \\
\hline Catarpe & Catarpe 2 & 1786 & $\begin{array}{c}\text { Torres-Rouff \& } \\
\text { Hubbe } 2013\end{array}$ & Beta- 251750 & Bone collagen & 750 & 40 & -9.2 & Yes & No & \\
\hline Catarpe & Catarpe 5 & 2392 & $\begin{array}{c}\text { Torres-Rouff \& } \\
\text { Hubbe } 2013\end{array}$ & X14966A & Bone collagen & 1123 & 44 & -13.3 & No & No & \\
\hline Catarpe & Catarpe 5 & 2385 & $\begin{array}{c}\text { Torres-Rouff \& } \\
\text { Hubbe } 2013\end{array}$ & X14965A & Bone collagen & 1083 & 47 & -13.2 & No & No & \\
\hline Conde Duque & Casa Parroquial & CP18 & $\begin{array}{l}\text { Torres-Rouff \& } \\
\text { Hubbe } 2013\end{array}$ & $\mathrm{X} 14962 \mathrm{~A}$ & Bone collagen & 1113 & 44 & -11.0 & No & No & \\
\hline Conde Duque & Casa Parroquial & $\mathrm{CP} 5$ & $\begin{array}{c}\text { Torres-Rouff \& } \\
\text { Hubbe } 2013\end{array}$ & X27412 & Bone collagen & 1091 & 46 & -12.9 & No & No & \\
\hline Conde Duque & Casa Parroquial & CP10 & $\begin{array}{c}\text { Torres-Rouff \& } \\
\text { Hubbe } 2013\end{array}$ & X27413 & Bone collagen & 1091 & 46 & -12.0 & No & No & \\
\hline Conde Duque & Casa Parroquial & $\mathrm{CP} 6^{*}$ & $\begin{array}{c}\text { Torres-Rouff \& } \\
\text { Hubbe } 2013\end{array}$ & X14961A & Bone collagen & 1067 & 44 & -12.8 & No & No & \\
\hline Conde Duque & Casa Parroquial & CP6* & $\begin{array}{c}\text { Torres-Rouff \& } \\
\text { Hubbe } 2013\end{array}$ & $\mathrm{X} 27414$ & Bone collagen & 1063 & 55 & -13.9 & No & No & \\
\hline Conde Duque & Casa Parroquial & $\mathrm{CP} 8$ & This study & AA111794 & Bone collagen & 1027 & 26 & -13.0 & No & No & \\
\hline Conde Duque & Casa Parroquial & CP9 & $\begin{array}{c}\text { Torres-Rouff \& } \\
\text { Hubbe } 2013\end{array}$ & $\mathrm{X} 27411$ & Bone collagen & 1018 & 55 & -13.1 & No & No & \\
\hline
\end{tabular}




\begin{tabular}{|c|c|c|c|c|c|c|c|c|c|c|c|}
\hline \multirow[b]{2}{*}{ Ayllu } & \multirow[b]{2}{*}{ Site } & \multirow[b]{2}{*}{$\begin{array}{c}\text { Sample ID } \\
\quad \text { (site \& } \\
\text { burial number) }\end{array}$} & \multirow[b]{2}{*}{ Source } & \multirow[b]{2}{*}{ Lab \# } & \multirow[b]{2}{*}{ Material } & \multirow[b]{2}{*}{ Radiocarbon age } & \multirow[b]{2}{*}{ (sd) } & \multirow[b]{2}{*}{${ }^{13} \mathrm{C}$} & \multicolumn{3}{|c|}{ Outlier? } \\
\hline & & & & & & & & & All ayllus & Inter-ayllu & Within ayllu \\
\hline Coyo & Coyo 3 & $\mathrm{~T} 57$ & $\begin{array}{c}\text { Torres-Rouff \& } \\
\text { Hubbe } 2013\end{array}$ & X30228 & Bone collagen & 1361 & 26 & -15.9 & No & No & No \\
\hline Coyo & Coyo 3 & $\mathrm{~T} 46$ & $\begin{array}{c}\text { Torres-Rouff \& } \\
\text { Hubbe } 2013\end{array}$ & X30227 & Bone collagen & 1329 & 25 & -13.6 & No & No & No \\
\hline Coyo & Coyo 3 & T51 & This study & AA111822 & Bone collagen & 1314 & 26 & -15.1 & No & No & No \\
\hline Coyo & Coyo 3 & $\mathrm{~T} 32$ & This study & AA111824 & Bone collagen & 1303 & 41 & -14.7 & No & No & No \\
\hline Coyo & Coyo 3 & $\mathrm{~T} 35$ & $\begin{array}{l}\text { Torres-Rouff \& } \\
\text { Hubbe } 2013\end{array}$ & X30224 & Bone collagen & 1302 & 28 & -13.2 & No & No & No \\
\hline Coyo & Coyo 3 & $\mathrm{~T} 13^{*}$ & $\begin{array}{l}\text { Torres-Rouff \& } \\
\text { Hubbe } 2013\end{array}$ & X30220 & Bone collagen & 1291 & 26 & -14.6 & No & No & No \\
\hline Coyo & Coyo 3 & $\mathrm{~T} 13 *$ & This study & AA111823 & Bone collagen & 1290 & 27 & -13.4 & No & No & No \\
\hline Coyo & Coyo 3 & T36 & $\begin{array}{c}\text { Torres-Rouff \& } \\
\text { Hubbe } 2013\end{array}$ & X30225 & Bone collagen & 1273 & 35 & -14.9 & No & No & No \\
\hline Coyo & Coyo 3 & $\mathrm{~T} 1 *$ & This study & AA107696 & Bone collagen & 1263 & 25 & -11.2 & No & No & No \\
\hline Coyo & Coyo 3 & $\mathrm{~T} 1 *$ & $\begin{array}{c}\text { Torres-Rouff \& } \\
\text { Hubbe } 2013\end{array}$ & X30219 & Bone collagen & 1263 & 25 & -11.2 & No & No & No \\
\hline Coyo & Coyo 3 & $\mathrm{~T} 18$ & This study & AA111820 & Bone collagen & 1258 & 26 & -12.4 & No & No & No \\
\hline Coyo & Coyo 3 & $\mathrm{~T} 23$ & $\begin{array}{c}\text { Torres-Rouff \& } \\
\text { Hubbe } 2013\end{array}$ & X30223 & Bone collagen & 1244 & 25 & -12.8 & No & No & No \\
\hline Coyo & Coyo 3 & $\mathrm{~T} 21$ & $\begin{array}{c}\text { Torres-Rouff \& } \\
\text { Hubbe } 2013\end{array}$ & X30222 & Bone collagen & 1232 & 26 & -12.1 & No & No & No \\
\hline Coyo & Coyo 3 & $\mathrm{~T} 28$ & This study & AA111821 & Bone collagen & 1231 & 26 & -12.9 & No & No & No \\
\hline Coyo & Coyo 3 & $\mathrm{~T} 16$ & $\begin{array}{c}\text { Torres-Rouff \& } \\
\text { Hubbe } 2013\end{array}$ & X30221 & Bone collagen & 1185 & 28 & -12.0 & No & No & Yes \\
\hline Coyo & Coyo 3 & $\mathrm{~T} 10$ & This study & AA111819 & Bone collagen & 1181 & 27 & -14.6 & No & No & Yes \\
\hline Coyo & Coyo Oriental & 4164 & This study & AA107733 & Bone collagen & 1449 & 26 & -14.4 & No & No & No \\
\hline Coyo & Coyo Oriental & 5317 & This study & AA107737 & Bone collagen & 1433 & 25 & -14.4 & No & No & No \\
\hline Coyo & Coyo Oriental & 5383 & Oakland 1992 & Beta-33858 & Textile & 1430 & 60 & $\mathrm{nr}$ & No & No & No \\
\hline Coyo & Coyo Oriental & 3973 & This study & AA107712 & Bone collagen & 1393 & 25 & -13.8 & No & No & No \\
\hline Coyo & Coyo Oriental & 4053 & This study & AA107718 & Bone collagen & 1388 & 25 & -16.2 & No & No & No \\
\hline
\end{tabular}




\begin{tabular}{|c|c|c|c|c|c|c|c|c|c|c|c|}
\hline \multirow[b]{2}{*}{ Ayllu } & \multirow[b]{2}{*}{ Site } & \multirow{2}{*}{$\begin{array}{c}\text { Sample ID } \\
\text { (site \& } \\
\text { burial number) }\end{array}$} & \multirow[b]{2}{*}{ Source } & \multirow[b]{2}{*}{ Lab \# } & \multirow[b]{2}{*}{ Material } & \multirow[b]{2}{*}{ Radiocarbon age } & \multirow[b]{2}{*}{ (sd) } & \multirow[b]{2}{*}{${ }^{13} \mathrm{C}$} & \multicolumn{3}{|c|}{ Outlier? } \\
\hline & & & & & & & & & All ayllus & Inter-ayllu & Within ayllu \\
\hline Coyo & Coyo Oriental & 3904 & This study & AA107706 & Bone collagen & 1378 & 26 & -15.1 & No & No & No \\
\hline Coyo & Coyo Oriental & 4052 & This study & AA107717 & Bone collagen & 1375 & 25 & -15.8 & No & No & No \\
\hline Coyo & Coyo Oriental & 4055 & This study & AA99873 & Bone collagen & 1375 & 46 & -15.0 & No & No & No \\
\hline Coyo & Coyo Oriental & 5334 & This study & AA107738 & Bone collagen & 1367 & 25 & -13.9 & No & No & No \\
\hline Coyo & Coyo Oriental & 4049 & This study & AA107716 & Bone collagen & 1362 & 25 & -14.6 & No & No & No \\
\hline Coyo & Coyo Oriental & 5300 & This study & AA107736 & Bone collagen & 1362 & 39 & -14.6 & No & No & No \\
\hline Coyo & Coyo Oriental & 4067 & This study & AA107722 & Bone collagen & 1361 & 25 & -15.4 & No & No & No \\
\hline Coyo & Coyo Oriental & 4109 & This study & AA107728 & Bone collagen & 1361 & 25 & -12.2 & No & No & No \\
\hline Coyo & Coyo Oriental & 3959 & This study & AA107711 & Bone collagen & 1360 & 25 & -15.0 & No & No & No \\
\hline Coyo & Coyo Oriental & 3956 & This study & AA107709 & Bone collagen & 1360 & 25 & -14.8 & No & No & No \\
\hline Coyo & Coyo Oriental & 4102 & This study & AA107727 & Bone collagen & 1355 & 25 & -14.9 & No & No & No \\
\hline Coyo & Coyo Oriental & 4003 & This study & AA99870 & Bone collagen & 1333 & 45 & -11.9 & No & No & No \\
\hline Coyo & Coyo Oriental & 4093 & This study & AA107725 & Bone collagen & 1330 & 25 & -13.3 & No & No & No \\
\hline Coyo & Coyo Oriental & 4077 & This study & AA107724 & Bone collagen & 1326 & 25 & -15.5 & No & No & No \\
\hline Coyo & Coyo Oriental & 5341 & Oakland 1992 & Beta-33856 & Textile & 1320 & 60 & $\mathrm{nr}$ & No & No & No \\
\hline Coyo & Coyo Oriental & 4020 & This study & AA107714 & Bone collagen & 1320 & 25 & -14.1 & No & No & No \\
\hline Coyo & Coyo Oriental & 3957 & This study & AA107710 & Bone collagen & 1315 & 25 & -15.7 & No & No & No \\
\hline Coyo & Coyo Oriental & 4069 & This study & AA107723 & Bone collagen & 1312 & 25 & -13.9 & No & No & No \\
\hline Coyo & Coyo Oriental & 5335 & This study & AA107739 & Bone collagen & 1311 & 25 & -15.5 & No & No & No \\
\hline Coyo & Coyo Oriental & 4012 & Oakland 1992 & Beta-33853 & Bone & 1310 & 70 & $\mathrm{nr}$ & No & No & No \\
\hline Coyo & Coyo Oriental & $4064 *$ & Oakland 1992 & Beta-33855 & Bone/muscle & 1310 & 80 & $\mathrm{nr}$ & No & No & No \\
\hline Coyo & Coyo Oriental & 4190 & This study & AA99868 & Bone collagen & 1305 & 45 & -14.6 & No & No & No \\
\hline Coyo & Coyo Oriental & 3948 & This study & AA107708 & Bone collagen & 1303 & 25 & -16.2 & No & No & No \\
\hline Coyo & Coyo Oriental & 4163 & This study & AA107732 & Bone collagen & 1301 & 26 & -15.9 & No & No & No \\
\hline Coyo & Coyo Oriental & 4193 & This study & AA107735 & Bone collagen & 1295 & 26 & -14.0 & No & No & No \\
\hline Coyo & Coyo Oriental & 4054 & This study & AA99869 & Bone collagen & 1293 & 45 & -13.7 & No & No & No \\
\hline Coyo & Coyo Oriental & 4080 & This study & AA99872 & Bone collagen & 1292 & 47 & -12.6 & No & No & No \\
\hline Coyo & Coyo Oriental & 4132 & This study & AA107729 & Bone collagen & 1280 & 25 & -14.5 & No & No & No \\
\hline Coyo & Coyo Oriental & 4098 & This study & AA107726 & Bone collagen & 1276 & 25 & -14.8 & No & No & No \\
\hline Coyo & Coyo Oriental & 5316 & This study & AA99866 & Bone collagen & 1265 & 46 & -12.8 & No & No & No \\
\hline
\end{tabular}


Sample ID

(site \&

\begin{tabular}{|c|c|c|c|c|c|c|c|c|c|c|c|}
\hline Ayllu & Site & burial number) & Source & Lab \# & Material & Radiocarbon age & (sd) & ${ }^{13} \mathrm{C}$ & All ayllus & Inter-ayllu & Within ayllu \\
\hline$\overline{\text { Coyo }}$ & Coyo Oriental & 4151 & This study & AA107731 & Bone collagen & 1264 & 25 & -13.3 & $\mathrm{No}$ & No & $\mathrm{No}$ \\
\hline Coyo & Coyo Oriental & 5308 & This study & AA99875 & Bone collagen & 1248 & 46 & -10.8 & No & No & No \\
\hline Coyo & Coyo Oriental & 4046 & This study & AA99867 & Bone collagen & 1247 & 45 & -13.4 & No & No & No \\
\hline Coyo & Coyo Oriental & 4147 & This study & AA107730 & Bone collagen & 1237 & 25 & -14.2 & No & No & No \\
\hline Coyo & Coyo Oriental & 4060 & This study & AA107720 & Bone collagen & 1218 & 25 & -13.4 & No & No & No \\
\hline Coyo & Coyo Oriental & 4059 & This study & AA107719 & Bone collagen & 1215 & 25 & -14.6 & No & No & No \\
\hline Coyo & Coyo Oriental & 3913 & This study & AA107707 & Bone collagen & 1213 & 25 & -13.9 & No & No & No \\
\hline Coyo & Coyo Oriental & 5343 & This study & AA 107740 & Bone collagen & 1196 & 25 & -14.2 & No & No & No \\
\hline Coyo & Coyo Oriental & 3984 & This study & AA107713 & Bone collagen & 1172 & 25 & -13.7 & No & No & No \\
\hline Coyo & Coyo Oriental & 4031 & This study & AA107715 & Bone collagen & 1157 & 25 & -12.8 & No & No & No \\
\hline Coyo & Coyo Oriental & 5347 & Oakland 1992 & Beta-33857 & Textile & 1155 & 80 & $\mathrm{nr}$ & No & No & No \\
\hline Coyo & Coyo Oriental & 4178 & This study & AA107734 & Bone collagen & 1137 & 25 & -12.7 & No & No & Yes \\
\hline Coyo & Coyo Oriental & $4064 *$ & This study & AA107721 & Bone collagen & 1126 & 25 & -11.8 & No & No & Yes \\
\hline Coyo & Coyo Oriental & 4158 & This study & AA99874 & Bone collagen & 1102 & 52 & -14.7 & No & Yes & Yes \\
\hline Coyo & Coyo Oriental & 4026 & Oakland 1992 & Beta-33854 & Muscle/skin & 1100 & 70 & $\mathrm{nr}$ & No & Yes & Yes \\
\hline Coyo & Coyo Oriental & 5345 & This study & AA107741 & Bone collagen & 1082 & 26 & -14.2 & No & Yes & Yes \\
\hline Coyo & Coyo Oriental & 4175 & This study & AA99871 & Bone collagen & 1020 & 44 & -14.4 & No & Yes & Yes \\
\hline Larache & Larache & 3803 & This study & AA111747 & Bone collagen & 1829 & 22 & -16.2 & Yes & No & \\
\hline Larache & Larache & 3797 & This study & AA111746 & Bone collagen & 1759 & 21 & -15.9 & Yes & No & \\
\hline Larache & Larache & 3802 & This study & AA111743 & Bone collagen & 1723 & 38 & -17.4 & No & No & \\
\hline Larache & Larache & 5056 & $\begin{array}{c}\text { Torres-Rouff \& } \\
\text { Hubbe } 2013\end{array}$ & X14967A & Bone collagen & 1667 & 45 & -17.1 & No & No & \\
\hline Larache & Larache & 1583 & This study & AA111744 & Bone collagen & 1363 & 21 & -15.3 & No & No & \\
\hline Larache & Larache & 357 & This study & AA111753 & Bone collagen & 1270 & 31 & -15.2 & No & No & \\
\hline Larache & Larache & 115 & This study & AA111757 & Bone collagen & 1230 & 26 & -13.6 & No & No & \\
\hline Larache & Larache & 356 & This study & AA111745 & Bone collagen & 1221 & 21 & -12.9 & No & No & \\
\hline Larache & Larache & 366 & This study & AA111755 & Bone collagen & 1202 & 21 & -14.3 & No & No & \\
\hline Larache & Larache & 124 & This study & AA111756 & Bone collagen & 1185 & 26 & -11.7 & No & No & \\
\hline Larache & Larache & 390 & This study & AA111748 & Bone collagen & 1120 & 20 & -11.0 & No & No & \\
\hline Larache & Larache & 358 & This study & AA111750 & Bone collagen & 1086 & 21 & -10.7 & No & No & \\
\hline
\end{tabular}




\begin{tabular}{|c|c|c|c|c|c|c|c|c|c|c|c|}
\hline \multirow[b]{2}{*}{ Ayllu } & \multirow[b]{2}{*}{ Site } & \multirow{2}{*}{$\begin{array}{c}\text { Sample ID } \\
\quad(\text { site \& } \\
\text { burial number })\end{array}$} & \multirow[b]{2}{*}{ Source } & \multirow[b]{2}{*}{ Lab \# } & \multirow[b]{2}{*}{ Material } & \multirow[b]{2}{*}{ Radiocarbon age } & \multirow[b]{2}{*}{ (sd) } & \multirow[b]{2}{*}{${ }^{13} \mathrm{C}$} & \multicolumn{3}{|c|}{ Outlier? } \\
\hline & & & & & & & & & All ayllus & Inter-ayllu & Within ayllu \\
\hline Larache & Larache & 125 & $\begin{array}{c}\text { Torres-Rouff \& } \\
\text { Hubbe } 2013\end{array}$ & X33243 & Bone collagen & 1076 & 25 & -14.8 & No & No & \\
\hline Larache & Larache & 3480 & This study & AA111754 & Bone collagen & 681 & 20 & -15.1 & Yes & Yes & \\
\hline Quitor & Quitor 1 & 3487 & $\begin{array}{c}\text { Torres-Rouff \& } \\
\text { Hubbe } 2013\end{array}$ & X14968A & Bone collagen & 956 & 44 & -15.6 & No & No & No \\
\hline Quitor & Quitor 2 & 3770 & $\begin{array}{c}\text { Torres-Rouff \& } \\
\text { Hubbe } 2013\end{array}$ & X14970A & Bone collagen & 1696 & 46 & -17.9 & No & No & No \\
\hline Quitor & Quitor 2 & 3716 & $\begin{array}{c}\text { Torres-Rouff \& } \\
\text { Hubbe } 2013\end{array}$ & Beta-251751 & Bone collagen & 1520 & 40 & -15.3 & No & No & No \\
\hline Quitor & Quitor 2 & 3684 & $\begin{array}{c}\text { Torres-Rouff \& } \\
\text { Hubbe } 2013\end{array}$ & X14969A & Bone collagen & 1491 & 46 & -17.3 & No & No & No \\
\hline Quitor & Quitor 2 & 3783 & $\begin{array}{c}\text { Torres-Rouff \& } \\
\text { Hubbe } 2013\end{array}$ & Beta-251752 & Bone collagen & 1310 & 40 & -13.6 & No & No & No \\
\hline Quitor & Quitor 2 & 1983:15 & Llagostera 1995 & Beta-53566 & unknown & 1190 & 50 & $\mathrm{nr}$ & No & No & No \\
\hline Quitor & Quitor 5 & $3397 *$ & Núñez 1976 & $\mathrm{I}-1205$ & unknown & 1750 & 80 & $\mathrm{nr}$ & No & No & No \\
\hline Quitor & Quitor 5 & 3347 & $\begin{array}{c}\text { Torres-Rouff \& } \\
\text { Hubbe } 2013\end{array}$ & $\mathrm{X} 27403$ & Bone collagen & 1742 & 40 & -15.9 & No & No & No \\
\hline Quitor & Quitor 5 & 3380 & This study & AA111791 & Bone collagen & 1735 & 26 & -17.1 & No & No & No \\
\hline Quitor & Quitor 5 & 2020 & This study & AA111792 & Bone collagen & 1720 & 26 & -16.7 & No & No & No \\
\hline Quitor & Quitor 5 & $3397 *$ & This study & AA111789 & Bone collagen & 1693 & 29 & -17.2 & No & No & No \\
\hline Quitor & Quitor 5 & 1942 & This study & AA111786 & Bone collagen & 1690 & 26 & -17.2 & No & No & No \\
\hline Quitor & Quitor 5 & 2021 & $\begin{array}{c}\text { Torres-Rouff \& } \\
\text { Hubbe } 2013\end{array}$ & X27402 & Bone collagen & 1673 & 40 & -16.7 & No & No & No \\
\hline Quitor & Quitor 5 & 1957 & $\begin{array}{c}\text { Torres-Rouff \& } \\
\text { Hubbe } 2013\end{array}$ & X27401 & Bone collagen & 1631 & 34 & -16.8 & No & No & No \\
\hline Quitor & Quitor 5 & 3066 & This study & AA111781 & Bone collagen & 1627 & 29 & -17.2 & No & No & No \\
\hline Quitor & Quitor 5 & 3394 & $\begin{array}{c}\text { Torres-Rouff \& } \\
\text { Hubbe } 2013\end{array}$ & X14974A & Bone collagen & 1623 & 46 & -17.1 & No & No & No \\
\hline Quitor & Quitor 5 & 2026 & This study & AA111785 & Bone collagen & 1575 & 26 & -15.8 & No & No & No \\
\hline
\end{tabular}


Table 1 (Continued)

Outlier?

\begin{tabular}{|c|c|c|c|c|c|c|c|c|c|c|c|}
\hline \multirow[b]{2}{*}{ Ayllu } & \multirow[b]{2}{*}{ Site } & \multirow[b]{2}{*}{$\begin{array}{c}\text { Sample ID } \\
\text { (site \& } \\
\text { burial number) }\end{array}$} & \multirow[b]{2}{*}{ Source } & \multirow[b]{2}{*}{ Lab \# } & \multirow[b]{2}{*}{ Material } & \multirow[b]{2}{*}{ Radiocarbon age } & \multirow[b]{2}{*}{ (sd) } & \multirow[b]{2}{*}{${ }^{13} \mathrm{C}$} & \multicolumn{3}{|c|}{ Outlier? } \\
\hline & & & & & & & & & All ayllus & Inter-ayllu & Within ayllu \\
\hline Quitor & Quitor 5 & 1964 & $\begin{array}{c}\text { Torres-Rouff \& } \\
\text { Hubbe } 2013\end{array}$ & X27404 & Bone collagen & 1543 & 34 & -17.7 & No & No & No \\
\hline Quitor & Quitor 5 & 2009 & $\begin{array}{c}\text { Torres-Rouff \& } \\
\text { Hubbe } 2013\end{array}$ & X14972A & Bone collagen & 1511 & 46 & -17.4 & No & No & No \\
\hline Quitor & Quitor 5 & 2109 & This study & AA111782 & Bone collagen & 1469 & 29 & -15.1 & No & No & No \\
\hline Quitor & Quitor 5 & 2055 & This study & AA111784 & Bone collagen & 1436 & 28 & -17.5 & No & No & No \\
\hline Quitor & Quitor 5 & 2100 & This study & AA111783 & Bone collagen & 1371 & 29 & -12.9 & No & No & No \\
\hline Quitor & Quitor 5 & 2179 & $\begin{array}{c}\text { Torres-Rouff \& } \\
\text { Hubbe } 2013\end{array}$ & X14973A & Bone collagen & 1338 & 45 & -11.1 & No & No & No \\
\hline Quitor & Quitor 5 & 1916 & This study & AA111779 & Bone collagen & 1326 & 28 & -15.3 & No & No & No \\
\hline Quitor & Quitor 5 & 2212 & This study & AA111787 & Bone collagen & 1243 & 26 & -12.8 & No & No & No \\
\hline Quitor & Quitor 5 & 2245 & This study & AA111780 & Bone collagen & 1191 & 28 & -11.5 & No & No & No \\
\hline Quitor & Quitor 5 & 2169 & $\begin{array}{c}\text { Torres-Rouff \& } \\
\text { Hubbe } 2013\end{array}$ & $\mathrm{X} 27405$ & Bone collagen & 1185 & 34 & -10.8 & No & No & No \\
\hline Quitor & Quitor 5 & 1921 & $\begin{array}{c}\text { Torres-Rouff \& } \\
\text { Hubbe } 2013\end{array}$ & $\mathrm{X} 14971 \mathrm{~A}$ & Bone collagen & 1164 & 44 & -13.5 & No & No & No \\
\hline Quitor & Quitor 6 & 2532 & Núñez 1976 & $\mathrm{Sa}-226$ & Wood & 1700 & 50 & $\mathrm{nr}$ & No & No & Yes \\
\hline Quitor & Quitor 6 & 3633 & $\begin{array}{c}\text { Torres-Rouff \& } \\
\text { Hubbe } 2013\end{array}$ & Beta-263470 & Bone collagen & 1490 & 40 & -16.9 & No & No & No \\
\hline Quitor & Quitor 6 & 2588 & $\begin{array}{c}\text { Torres-Rouff \& } \\
\text { Hubbe } 2013\end{array}$ & Beta-263468 & Bone collagen & 1290 & 40 & -15.6 & No & No & No \\
\hline Quitor & Quitor 6 & 2928 & $\begin{array}{c}\text { Torres-Rouff \& } \\
\text { Hubbe } 2013\end{array}$ & Beta-263469 & Bone collagen & 1180 & 40 & -12.5 & No & No & No \\
\hline Quitor & Quitor 6 & 2529 & $\begin{array}{c}\text { Torres-Rouff \& } \\
\text { Hubbe } 2013\end{array}$ & Beta-263467 & Bone collagen & 1050 & 40 & -13.8 & No & No & No \\
\hline Quitor & Quitor 6 Tardío & T27 C871 & This study & AA111764 & Bone collagen & 1508 & 26 & -17.3 & No & No & No \\
\hline Quitor & Quitor 6 Tardío & $\mathrm{T} 28 \mathrm{C} 876$ & This study & AA111768 & Bone collagen & 1490 & 27 & -16.6 & No & No & No \\
\hline Quitor & Quitor 6 Tardío & $\mathrm{T} 17 \mathrm{C} 222 / 223$ & This study & AA111770 & Bone collagen & 1415 & 27 & -13.7 & No & No & No \\
\hline Quitor & Quitor 6 Tardío & T28 C877 & This study & AA111769 & Bone collagen & 1403 & 26 & -14.9 & No & No & No \\
\hline Quitor & Quitor 6 Tardío & T14 C469/470 & This study & AA111763 & Bone collagen & 1259 & 27 & -14.1 & No & No & No \\
\hline
\end{tabular}




\begin{tabular}{|c|c|c|c|c|c|c|c|c|c|c|c|}
\hline \multirow[b]{2}{*}{ Ayllu } & \multirow[b]{2}{*}{ Site } & \multirow{2}{*}{$\begin{array}{c}\text { Sample ID } \\
\quad \text { (site \& } \\
\text { burial number) }\end{array}$} & \multirow[b]{2}{*}{ Source } & \multirow[b]{2}{*}{ Lab \# } & \multirow[b]{2}{*}{ Material } & \multirow[b]{2}{*}{ Radiocarbon age } & \multirow[b]{2}{*}{ (sd) } & \multirow[b]{2}{*}{${ }^{13} \mathrm{C}$} & \multicolumn{3}{|c|}{ Outlier? } \\
\hline & & & & & & & & & All ayllus & Inter-ayllu & Within ayllu \\
\hline Quitor & Quitor 6 Tardío & T54 C685 & This study & AA111774 & Bone collagen & 1147 & 26 & -11.6 & No & No & No \\
\hline Quitor & Quitor 6 Tardío & T55 C694 & This study & AA111767 & Bone collagen & 1146 & 26 & -14.3 & No & No & No \\
\hline Quitor & Quitor 6 Tardío & T8 C80/81 & This study & AA111772 & Bone collagen & 1072 & 26 & -14.9 & No & No & No \\
\hline Quitor & Quitor 6 Tardío & T9 C88/89 & This study & AA111765 & Bone collagen & 1062 & 26 & -14.0 & No & No & No \\
\hline Quitor & Quitor 6 Tardío & T10 C104 & This study & AA111771 & Bone collagen & 1054 & 27 & -13.8 & No & No & No \\
\hline Quitor & Quitor 6 Tardío & T36 & Costa 1988 & Beta-11208 & Textile & 1030 & 70 & $\mathrm{nr}$ & No & No & No \\
\hline Quitor & Quitor 6 Tardío & T38 C943 & This study & AA111766 & Bone collagen & 974 & 26 & -14.1 & No & No & No \\
\hline Quitor & Quitor 6 Tardío & T50 & Costa 1988 & Beta-9349 & Wood & 810 & 80 & $\mathrm{nr}$ & Yes & Yes & Yes \\
\hline Quitor & Quitor 6 Tardío & $\mathrm{T} 35$ & Costa 1988 & Beta-11207 & Wood & 710 & 70 & $\mathrm{nr}$ & Yes & Yes & Yes \\
\hline Quitor & Quitor 8 & 3227 & This study & AA111776 & Bone collagen & 1657 & 27 & -16.1 & No & No & No \\
\hline Quitor & Quitor 8 & 3202 & This study & AA111777 & Bone collagen & 1651 & 31 & -16.9 & No & No & No \\
\hline Quitor & Quitor 8 & 3146 & This study & AA111775 & Bone collagen & 1597 & 31 & -16.5 & No & No & No \\
\hline Quitor & Quitor 8 & 3145 & $\begin{array}{c}\text { Torres-Rouff \& } \\
\text { Hubbe } 2013\end{array}$ & Beta-251753 & Bone collagen & 1510 & 40 & -17.3 & No & No & No \\
\hline Quitor & Quitor 8 & 3226 & $\begin{array}{l}\text { Torres-Rouff \& } \\
\text { Hubbe } 2013\end{array}$ & Beta-251754 & Bone collagen & 1450 & 40 & -16.4 & No & No & No \\
\hline Quitor & Quitor 9 & $3236^{*}$ & This study & AA111761 & Bone collagen & 1148 & 26 & -12.2 & No & No & No \\
\hline Quitor & Quitor 9 & 3237 & This study & AA111760 & Bone collagen & 1090 & 25 & -12.8 & No & No & No \\
\hline Quitor & Quitor 9 & 3249 & This study & AA111762 & Bone collagen & 1077 & 26 & -2.2 & No & No & No \\
\hline Quitor & Quitor 9 & 3251 & $\begin{array}{l}\text { Torres-Rouff \& } \\
\text { Hubbe } 2013\end{array}$ & X14976A & Bone collagen & 1068 & 44 & -13.7 & No & No & No \\
\hline Quitor & Quitor 9 & $3236^{*}$ & Núñez 1976 & $\mathrm{I}-1205$ & unknown & 900 & 80 & $\mathrm{nr}$ & No & No & No \\
\hline Sequitor & $\begin{array}{l}\text { Sequitor } \\
\text { Alambrado }\end{array}$ & 1068 & $\begin{array}{l}\text { Torres-Rouff \& } \\
\text { Hubbe } 2013\end{array}$ & Beta-251746 & Bone collagen & 1680 & 40 & -17.5 & No & No & \\
\hline Sequitor & $\begin{array}{l}\text { Sequitor } \\
\quad \text { Alambrado }\end{array}$ & 1043 & $\begin{array}{l}\text { Torres-Rouff \& } \\
\text { Hubbe } 2013\end{array}$ & Beta-263472 & Bone collagen & 1680 & 40 & -15.9 & No & No & \\
\hline Sequitor & $\begin{array}{l}\text { Sequitor } \\
\text { Alambrado }\end{array}$ & 1062 & $\begin{array}{l}\text { Torres-Rouff \& } \\
\text { Hubbe } 2013\end{array}$ & Beta-251745 & Bone collagen & 1600 & 40 & -15.9 & No & No & \\
\hline Solcor & Solcor 3 & T54 C2071 & $\begin{array}{l}\text { Torres-Rouff \& } \\
\text { Hubbe } 2013\end{array}$ & X30273 & Bone collagen & 1649 & 26 & -16.6 & No & Yes & Yes \\
\hline
\end{tabular}


Table 1 (Continued)

Outlier?

Sample ID

(site \&

\begin{tabular}{|c|c|c|c|c|c|c|c|c|c|c|c|}
\hline Ayllu & Site & burial number) & Source & Lab \# & Material & Radiocarbon age & (sd) & ${ }^{13} \mathrm{C}$ & All ayllus & Inter-ayllu & Within ayllu \\
\hline Solcor & Solcor 3 & T60 C2342 & $\begin{array}{c}\text { Torres-Rouff \& } \\
\text { Hubbe } 2013\end{array}$ & X30274 & Bone collagen & 1641 & 26 & -16.8 & No & Yes & Yes \\
\hline Solcor & Solcor 3 & T56 C3070 & This study & AA111809 & Bone collagen & 1521 & 27 & -15.4 & No & No & Yes \\
\hline Solcor & Solcor 3 & T117 C13156* & $\begin{array}{l}\text { Llagostera et al. } \\
1988\end{array}$ & Beta-27192 & unknown & 1470 & 80 & $\mathrm{nr}$ & No & No & No \\
\hline Solcor & Solcor 3 & T23 C1536 & $\begin{array}{l}\text { Llagostera et al. } \\
1988\end{array}$ & Beta-27572 & unknown & 1470 & 60 & $\mathrm{nr}$ & No & No & No \\
\hline Solcor & Solcor 3 & T75 C2607 & This study & AA111805 & Bone collagen & 1461 & 27 & -15.6 & No & No & No \\
\hline Solcor & Solcor 3 & T29 C1666 & This study & AA111808 & Bone collagen & 1430 & 27 & -16.3 & No & No & No \\
\hline Solcor & Solcor 3 & T27 C1628 & This study & AA111800 & Bone collagen & 1392 & 27 & -15.6 & No & No & No \\
\hline Solcor & Solcor 3 & T139 & This study & AA111796 & Bone collagen & 1391 & 26 & -16.3 & No & No & No \\
\hline Solcor & Solcor 3 & T107 C13118* & $\begin{array}{l}\text { Llagostera et al. } \\
1988\end{array}$ & Beta-22461 & unknown & 1380 & 60 & $\mathrm{nr}$ & No & No & No \\
\hline Solcor & Solcor 3 & T6 C1080 & This study & AA111815 & Bone collagen & 1373 & 28 & -15.4 & No & No & No \\
\hline Solcor & Solcor 3 & T70 C2514 & $\begin{array}{c}\text { Torres-Rouff \& } \\
\text { Hubbe } 2013\end{array}$ & X30277 & Bone collagen & 1366 & 25 & -15.0 & No & No & No \\
\hline Solcor & Solcor 3 & T60 C2341 & $\begin{array}{c}\text { Torres-Rouff \& } \\
\text { Hubbe } 2013\end{array}$ & X30275 & Bone collagen & 1364 & 25 & -15.0 & No & No & No \\
\hline Solcor & Solcor 3 & T116 C13126 & This study & AA111814 & Bone collagen & 1359 & 27 & -16.9 & No & No & No \\
\hline Solcor & Solcor 3 & T27 C1629 & $\begin{array}{c}\text { Torres-Rouff \& } \\
\text { Hubbe } 2013\end{array}$ & X30292 & Bone collagen & 1357 & 25 & -16.0 & No & No & No \\
\hline Solcor & Solcor 3 & T30 C1683 & This study & AA111799 & Bone collagen & 1355 & 27 & -15.8 & No & No & No \\
\hline Solcor & Solcor 3 & T79 C2762 & This study & AA111817 & Bone collagen & 1350 & 27 & -15.4 & No & No & No \\
\hline Solcor & Solcor 3 & T70 C2513 & $\begin{array}{c}\text { Torres-Rouff \& } \\
\text { Hubbe } 2013\end{array}$ & X30276 & Bone collagen & 1347 & 25 & -14.7 & No & No & No \\
\hline Solcor & Solcor 3 & $106 \mathrm{C} 13177$ & This study & AA111804 & Bone collagen & 1322 & 26 & -15.2 & No & No & No \\
\hline Solcor & Solcor 3 & T16 C3061 & $\begin{array}{c}\text { Torres-Rouff \& } \\
\text { Hubbe } 2013\end{array}$ & X30270 & Bone collagen & 1322 & 25 & -16.1 & No & No & No \\
\hline Solcor & Solcor 3 & T103 C3599 & This study & AA111795 & Bone collagen & 1316 & 27 & -12.6 & No & No & No \\
\hline
\end{tabular}




\begin{tabular}{|c|c|c|c|c|c|c|c|c|c|c|c|}
\hline \multirow[b]{2}{*}{ Ayllu } & \multirow[b]{2}{*}{ Site } & \multirow{2}{*}{$\begin{array}{c}\text { Sample ID } \\
\text { (site \& } \\
\text { burial number) }\end{array}$} & \multirow[b]{2}{*}{ Source } & \multirow[b]{2}{*}{ Lab \# } & \multirow[b]{2}{*}{ Material } & \multirow[b]{2}{*}{ Radiocarbon age } & \multirow[b]{2}{*}{ (sd) } & \multirow[b]{2}{*}{${ }^{13} \mathrm{C}$} & \multicolumn{3}{|c|}{ Outlier? } \\
\hline & & & & & & & & & All ayllus & Inter-ayllu & Within ayllu \\
\hline Solcor & Solcor 3 & T112 C13111* & $\begin{array}{l}\text { Torres-Rouff \& } \\
\text { Hubbe } 2013\end{array}$ & X30267 & Bone collagen & 1312 & 26 & -14.8 & No & No & No \\
\hline Solcor & Solcor 3 & T107 C13118* & $\begin{array}{l}\text { Torres-Rouff \& } \\
\text { Hubbe } 2013\end{array}$ & X30266 & Bone collagen & 1302 & 25 & -14.7 & No & No & No \\
\hline Solcor & Solcor 3 & T126 & This study & AA111818 & Bone collagen & 1298 & 27 & -13.9 & No & No & No \\
\hline Solcor & Solcor 3 & T115 C3610 & This study & AA111802 & Bone collagen & 1292 & 27 & -13.9 & No & No & No \\
\hline Solcor & Solcor 3 & T78 C2699 & This study & AA111813 & Bone collagen & 1289 & 27 & -14.8 & No & No & No \\
\hline Solcor & Solcor 3 & T115 C3609 & This study & AA111807 & Bone collagen & 1271 & 27 & -15.1 & No & No & No \\
\hline Solcor & Solcor 3 & $\mathrm{~T} 2$ & $\begin{array}{l}\text { Llagostera et al. } \\
1988\end{array}$ & Beta-27191 & unknown & 1270 & 90 & $\mathrm{nr}$ & No & No & No \\
\hline Solcor & Solcor 3 & T117 C13156* & $\begin{array}{c}\text { Torres-Rouff \& } \\
\text { Hubbe } 2013\end{array}$ & X30268 & Bone collagen & 1270 & 25 & -14.0 & No & No & No \\
\hline Solcor & Solcor 3 & T113 C13120 & This study & AA111797 & Bone collagen & 1266 & 26 & -14.3 & No & No & No \\
\hline Solcor & Solcor 3 & T115 C3611 & This study & AA111798 & Bone collagen & 1242 & 26 & -14.3 & No & No & No \\
\hline Solcor & Solcor 3 & T111 C3604 & This study & AA111812 & Bone collagen & 1224 & 30 & -13.0 & No & No & No \\
\hline Solcor & Solcor 3 & T101 C3597 & $\begin{array}{c}\text { Torres-Rouff \& } \\
\text { Hubbe } 2013\end{array}$ & X30265R & Bone collagen & 1223 & 25 & -12.0 & No & No & No \\
\hline Solcor & Solcor 3 & T111 C3605 & This study & AA111803 & Bone collagen & 1215 & 26 & -13.2 & No & No & No \\
\hline Solcor & Solcor 3 & T30 C1871 & $\begin{array}{c}\text { Torres-Rouff \& } \\
\text { Hubbe } 2013\end{array}$ & X30272 & Bone collagen & 1214 & 25 & -12.2 & No & No & No \\
\hline Solcor & Solcor 3 & T111 C3606 & This study & AA111806 & Bone collagen & 1205 & 27 & -13.7 & No & No & No \\
\hline Solcor & Solcor 3 & T98 C3593a & This study & AA111816 & Bone collagen & 1202 & 27 & -13.0 & No & No & No \\
\hline Solcor & Solcor 3 & T132 & $\begin{array}{c}\text { Torres-Rouff \& } \\
\text { Hubbe } 2013\end{array}$ & X30269 & Bone collagen & 1202 & 28 & -12.4 & No & No & No \\
\hline Solcor & Solcor 3 & T112 C13111* & $\begin{array}{c}\text { Torres et al. } \\
1991\end{array}$ & Beta-32447 & Skin/muscle & 1170 & 60 & $\mathrm{nr}$ & No & No & No \\
\hline Solcor & Solcor 3 & T24 C1558 & $\begin{array}{l}\text { Torres-Rouff \& } \\
\text { Hubbe } 2013\end{array}$ & Beta-305870 & Bone collagen & 1160 & 30 & -15.9 & No & No & No \\
\hline Solcor & Solcor 3 & T32 C1737 & This study & AA111801 & Bone collagen & 1156 & 26 & -13.1 & No & No & No \\
\hline
\end{tabular}


Table 1 (Continued)

Outlier?

\begin{tabular}{|c|c|c|c|c|c|c|c|c|c|c|c|}
\hline \multirow[b]{2}{*}{ Ayllu } & \multirow[b]{2}{*}{ Site } & \multirow[b]{2}{*}{$\begin{array}{c}\text { Sample ID } \\
\quad \text { (site \& } \\
\text { burial number) }\end{array}$} & \multirow[b]{2}{*}{ Source } & \multirow[b]{2}{*}{ Lab \# } & \multirow[b]{2}{*}{ Material } & \multirow[b]{2}{*}{ Radiocarbon age } & \multirow[b]{2}{*}{ (sd) } & \multirow[b]{2}{*}{${ }^{13} \mathrm{C}$} & \multicolumn{3}{|c|}{ Outlier? } \\
\hline & & & & & & & & & All ayllus & Inter-ayllu & Within ayllu \\
\hline Solcor & Solcor 3 & T8 C11161A & $\begin{array}{c}\text { Torres-Rouff \& } \\
\text { Hubbe } 2013\end{array}$ & Beta-305869 & Bone collagen & 1080 & 30 & -17.6 & No & No & Yes \\
\hline Solcor & Solcor 3 & N/A & $\begin{array}{l}\text { Llagostera et al. } \\
1988\end{array}$ & Beta-27573 & unknown & 1040 & 50 & $\mathrm{nr}$ & No & No & Yes \\
\hline Solcor & $\begin{array}{c}\text { Solcor Nueva } \\
\text { Población }\end{array}$ & 4791 & This study & AA111826 & Bone collagen & 1051 & 27 & -13.6 & No & No & No \\
\hline Solcor & $\begin{array}{c}\text { Solcor Nueva } \\
\text { Población }\end{array}$ & 4789 & This study & AA111827 & Bone collagen & 1041 & 27 & -15.7 & No & No & No \\
\hline Solcor & $\begin{array}{c}\text { Solcor Nueva } \\
\text { Población }\end{array}$ & 4778 & This study & AA111825 & Bone collagen & 1003 & 28 & -14.5 & No & No & No \\
\hline Solcor & Solcor Plaza & 5093 & $\begin{array}{c}\text { Torres-Rouff \& } \\
\text { Hubbe } 2013\end{array}$ & X30290 & Bone collagen & 1071 & 25 & -14.7 & No & No & No \\
\hline Solcor & Solcor Plaza & 1377 & $\begin{array}{c}\text { Torres-Rouff \& } \\
\text { Hubbe } 2013\end{array}$ & X30284 & Bone collagen & 1057 & 27 & -13.0 & No & No & No \\
\hline Solcor & Solcor Plaza & 1286 & $\begin{array}{c}\text { Torres-Rouff \& } \\
\text { Hubbe } 2013\end{array}$ & X30293 & Bone collagen & 1041 & 25 & -15.2 & No & No & No \\
\hline Solcor & Solcor Plaza & 1246 & This study & AA107759 & Bone collagen & 1036 & 29 & -13.2 & No & No & No \\
\hline Solcor & Solcor Plaza & 1244 & $\begin{array}{c}\text { Torres-Rouff \& } \\
\text { Hubbe } 2013\end{array}$ & X30281 & Bone collagen & 1031 & 28 & -14.2 & No & No & No \\
\hline Solcor & Solcor Plaza & 1243 & $\begin{array}{c}\text { Torres-Rouff \& } \\
\text { Hubbe } 2013\end{array}$ & X30280 & Bone collagen & 1028 & 25 & -14.6 & No & No & No \\
\hline Solcor & Solcor Plaza & 1391 & $\begin{array}{c}\text { Torres-Rouff \& } \\
\text { Hubbe } 2013\end{array}$ & X30288 & Bone collagen & 1001 & 25 & -13.9 & No & No & No \\
\hline Solcor & Solcor Plaza & 629 & $\begin{array}{c}\text { Torres-Rouff \& } \\
\text { Hubbe } 2013\end{array}$ & X30291 & Bone collagen & 997 & 25 & -14.9 & No & No & No \\
\hline Solcor & Solcor Plaza & 1394 & $\begin{array}{c}\text { Torres-Rouff \& } \\
\text { Hubbe } 2013\end{array}$ & X30289 & Bone collagen & 990 & 25 & -14.8 & No & No & No \\
\hline Solcor & Solcor Plaza & 1241 & $\begin{array}{c}\text { Torres-Rouff \& } \\
\text { Hubbe } 2013\end{array}$ & X14978A & Bone collagen & 987 & 44 & -14.8 & No & No & No \\
\hline
\end{tabular}




\begin{tabular}{|c|c|c|c|c|c|c|c|c|c|c|c|}
\hline \multirow[b]{2}{*}{ Ayllu } & \multirow[b]{2}{*}{ Site } & \multirow{2}{*}{$\begin{array}{c}\text { Sample ID } \\
\text { (site \& } \\
\text { burial number) }\end{array}$} & \multirow[b]{2}{*}{ Source } & \multirow[b]{2}{*}{ Lab \# } & \multirow[b]{2}{*}{ Material } & \multirow[b]{2}{*}{ Radiocarbon age } & \multirow[b]{2}{*}{ (sd) } & \multirow[b]{2}{*}{${ }^{13} \mathrm{C}$} & \multicolumn{3}{|c|}{ Outlier? } \\
\hline & & & & & & & & & All ayllus & Inter-ayllu & Within ayllu \\
\hline Solcor & Solcor Plaza & 1381 & $\begin{array}{l}\text { Torres-Rouff \& } \\
\text { Hubbe } 2013\end{array}$ & X30286 & Bone collagen & 951 & 25 & -13.9 & No & No & No \\
\hline Solcor & Solcor Plaza & 1379 & $\begin{array}{c}\text { Torres-Rouff \& } \\
\text { Hubbe } 2013\end{array}$ & X30285 & Bone collagen & 894 & 26 & -12.3 & Yes & Yes & Yes \\
\hline Solor & Solor 3 & 991 & $\begin{array}{c}\text { Torres-Rouff \& } \\
\text { Hubbe } 2013\end{array}$ & X14981A & Bone collagen & 1859 & 47 & -17.8 & Yes & No & \\
\hline Solor & Solor 3 & 983 & $\begin{array}{l}\text { Torres-Rouff \& } \\
\text { Hubbe } 2013\end{array}$ & X14980A & Bone collagen & 1616 & 46 & -15.8 & No & No & \\
\hline Solor & Solor 4 & cementerio N/A (1) & Núñez 1976 & Gr-N-4124 & Wood & 970 & 75 & $\mathrm{nr}$ & No & No & \\
\hline Solor & Solor 4 & cementerio N/A (2) & Núñez 1976 & Gr-N-4125 & Wood & 770 & 65 & $\mathrm{nr}$ & Yes & No & \\
\hline Solor & Solor 6 & between tombs N/A & Núñez 1976 & Sa-109 & Wood & 1650 & 50 & $\mathrm{nr}$ & No & No & \\
\hline Solor & Solor Vilama 3 & 300 & $\begin{array}{c}\text { Torres-Rouff \& } \\
\text { Hubbe } 2013\end{array}$ & Beta-293924 & Bone collagen & 1280 & 30 & -10.7 & No & No & \\
\hline Solor & Solor Vilama 3 & 308 & $\begin{array}{c}\text { Torres-Rouff \& } \\
\text { Hubbe } 2013\end{array}$ & X14983A & Bone collagen & 1125 & 45 & -14.6 & No & No & \\
\hline Solor & Solor Vilama 3 & 265 & $\begin{array}{c}\text { Torres-Rouff \& } \\
\text { Hubbe } 2013\end{array}$ & X14982A & Bone collagen & 1076 & 45 & -13.7 & No & No & \\
\hline Tchecar & $\begin{array}{l}\text { Tchecar Túmulo } \\
\text { Sur }\end{array}$ & 686 & $\begin{array}{l}\text { Torres-Rouff \& } \\
\text { Hubbe } 2013\end{array}$ & X27407 & Bone collagen & 1429 & 38 & -14.3 & No & No & \\
\hline Tchecar & $\begin{array}{l}\text { Tchecar Túmulo } \\
\text { Sur }\end{array}$ & 815 & $\begin{array}{c}\text { Torres-Rouff \& } \\
\text { Hubbe } 2013\end{array}$ & X27406 & Bone collagen & 1296 & 33 & -13.6 & No & No & \\
\hline Tchecar & $\begin{array}{l}\text { Tchecar Túmulo } \\
\text { Sur }\end{array}$ & 824 & $\begin{array}{l}\text { Torres-Rouff \& } \\
\text { Hubbe } 2013\end{array}$ & Beta-263475 & Bone collagen & 1240 & 40 & -13.7 & No & No & \\
\hline Tchecar & $\begin{array}{l}\text { Tchecar Túmulo } \\
\text { Sur }\end{array}$ & 838 & $\begin{array}{c}\text { Torres-Rouff \& } \\
\text { Hubbe } 2013\end{array}$ & Beta-293928 & Bone collagen & 1190 & 30 & -13.2 & No & No & \\
\hline Tchecar & $\begin{array}{l}\text { Tchecar Túmulo } \\
\text { Sur }\end{array}$ & 807 & $\begin{array}{l}\text { Torres-Rouff \& } \\
\text { Hubbe } 2013\end{array}$ & X27409 & Bone collagen & 1137 & 46 & -13.0 & No & No & \\
\hline Tchecar & $\begin{array}{l}\text { Tchecar Túmulo } \\
\text { Sur }\end{array}$ & 680 & $\begin{array}{c}\text { Torres-Rouff \& } \\
\text { Hubbe } 2013\end{array}$ & X27408 & Bone collagen & 1091 & 46 & -13.7 & No & No & \\
\hline
\end{tabular}


Table 1 (Continued)

Outlier?

\begin{tabular}{|c|c|c|c|c|c|c|c|c|c|c|c|}
\hline Ayllu & Site & $\begin{array}{c}\text { Sample ID } \\
\quad \text { (site \& } \\
\text { burial number) }\end{array}$ & Source & Lab \# & Material & Radiocarbon age & (sd) & ${ }^{13} \mathrm{C}$ & All ayllus & Inter-ayllu & Within ayllu \\
\hline Tchecar & $\begin{array}{l}\text { Tchecar Túmulo } \\
\text { Sur }\end{array}$ & 650 & $\begin{array}{c}\text { Torres-Rouff \& } \\
\text { Hubbe } 2013\end{array}$ & Beta-263473 & Bone collagen & 1090 & 40 & -12.3 & No & No & \\
\hline Tchecar & $\begin{array}{l}\text { Tchecar Túmulo } \\
\text { Sur }\end{array}$ & 1158 & $\begin{array}{c}\text { Torres-Rouff \& } \\
\text { Hubbe } 2013\end{array}$ & $\mathrm{X} 27410$ & Bone collagen & 1018 & 46 & -13.5 & No & No & \\
\hline Tchecar & $\begin{array}{l}\text { Tchecar Túmulo } \\
\text { Sur }\end{array}$ & 806 & $\begin{array}{c}\text { Torres-Rouff \& } \\
\text { Hubbe } 2013\end{array}$ & Beta-263474 & Bone collagen & 960 & 40 & -13.4 & No & No & \\
\hline Tulor & Tulor 1 & Circle 2 & Baron 1986 & $?$ & Charcoal? & 1885 & 95 & $\mathrm{nr}$ & Yes & No & \\
\hline Tulor & Tulor 1 & Recinto $3 \mathrm{C}$ & $\begin{array}{l}\text { Llagostera et al. } \\
1984\end{array}$ & P-3351 & Charcoal & 1850 & 60 & $\mathrm{nr}$ & Yes & No & \\
\hline Tulor & Tulor 1 & Sector 7 nivel 9 & Baron 1986 & $?$ & Charcoal? & 1775 & 250 & $\mathrm{nr}$ & Yes & No & \\
\hline Tulor & Tulor 1 & Sector 7 nivel 2 & Baron 1986 & $?$ & Charcoal? & 1690 & 170 & $\mathrm{nr}$ & No & No & \\
\hline Yaye & Yaye 1 & 5498 & $\begin{array}{c}\text { Torres-Rouff \& } \\
\text { Hubbe } 2013\end{array}$ & Beta-251756 & Bone collagen & 1100 & 40 & -13.9 & No & No & \\
\hline Yaye & Yaye 1 & 5494 & $\begin{array}{c}\text { Torres-Rouff \& } \\
\text { Hubbe } 2013\end{array}$ & Beta-251755 & Bone collagen & 920 & 40 & -11.7 & Yes & No & \\
\hline Yaye & Yaye 2 & 3309 & $\begin{array}{c}\text { Torres-Rouff \& } \\
\text { Hubbe } 2013\end{array}$ & Beta-251757 & Bone collagen & 1300 & 40 & -12.6 & No & No & \\
\hline Yaye & Yaye 2 & 3417 & $\begin{array}{c}\text { Torres-Rouff \& } \\
\text { Hubbe } 2013\end{array}$ & Beta-251758 & Bone collagen & 1040 & 40 & -13.2 & No & No & \\
\hline Yaye & Yaye 3 & 1573 & $\begin{array}{c}\text { Torres-Rouff \& } \\
\text { Hubbe } 2013\end{array}$ & Beta-251759 & Bone collagen & 1180 & 40 & -15.0 & No & No & \\
\hline Yaye & Yaye 4 & 1545 & $\begin{array}{c}\text { Torres-Rouff \& } \\
\text { Hubbe } 2013\end{array}$ & Beta-251760 & Bone collagen & 1170 & 40 & -11.0 & No & No & \\
\hline
\end{tabular}


The next model iteration (inter-ayllu) was performed on an ayllu-by-ayllu basis to establish phases of use/occupation of each ayllu. Prior to phase modeling, outliers (defined as above) were removed on an ayllu-by-ayllu basis, reducing the overall sample size for this analysis to 234 (removed outliers noted in Table 1). Calibration and phase modeling was performed as above using OxCal v 4.3.2 and the SHCall3 curve. In the case of the ayllu-level modeling, we employed the "Overlapping" phase option of OxCal, as there are no reliable independent priors relating to the succession of ayllu occupation. As before, no marine reservoir was employed in calibration and two sigma $(95.4 \%)$ ranges are presented for both individual samples and phase boundaries in all resulting tables and figures. Model and individual sample agreement indices for this iteration are presented in Table S2. Samples with agreement indices falling below the recommended cutoff value of 60\% (Bronk Ramsey 1995, 2009) were removed and the model subsequently re-run.

Finally, we further investigated the use-life of cemeteries internal to the three ayllus for which we have the largest and most representative samples: Coyo, Quitor, and Solcor (Figure 1). This iteration of modeling (intra-ayllu) was intended to assess the degree of contemporaneity of cemetery use internal to each of these geographical/social units. We chose to focus on these three ayllus (comprising 10 cemeteries) as they present the largest and best explored putatively Middle Period cemeteries in our sample. Importantly, analyzing multiple cemeteries from the same ayllu also allows us the ability to explore internal social differentiation while controlling for temporal distinctions. Varied excavation strategies over the decades have resulted in diverse records, however, together these 10 cemeteries have large sample sizes, high quality preservation, well-documented mortuary contexts, and include metals, elaborate textiles, ritual paraphernalia, and objects from varied foreign groups (Llagostera et al. 1988; Oakland 1992; Llagostera 1995; Torres-Rouff 2008; Figueroa et al. 2013). As such, these cemeteries provide a thorough view into the Middle Period in the San Pedro de Atacama oases.

The dates included for each cemetery were again trimmed for outliers following the procedure employed above, resulting in a total of 62 dates from two cemeteries in Coyo, 54 from five cemeteries in Quitor, and 52 from three cemeteries in Solcor (Table 1). Each ayllu was modeled independently following the same procedures identified above (OxCal v 4.3.2 and SHCal13 curve, "Overlapping" phase option, no priors, no marine reservoir, two sigma ranges, and reporting of any/all models/samples with agreement indices falling below the recommended cutoff value of $60 \%$, with subsequent removal and re-running of model without those samples as noted below). Model and individual sample agreement indices for this iteration are presented in Tables S3-S5.

\section{RESULTS}

We present here the results of our analyses at each of the three scales discussed above. Initially we consider the pattern revealed by integrating all the dates for the oases to explore broader patterns of occupation. Subsequently, we move into a presentation and discussion of the results between ayllus. Finally, we conclude with a deeper exploration of the three ayllus for which we have the most dates, allowing us to consider broader questions of internal variation and cemetery use across the oases. In all cases, we refer to the Boundary Start and End ages from the various iterations of the OxCal models as the de facto beginnings and ends, respectively, of the use-life of the respective ayllus and cemeteries. 




Figure 2 Structure of all ayllu model.

\section{All Ayllus}

The structure of the model that includes all the available dates for the San Pedro oases is presented in Figure 2, with details of phase boundaries and individual dates presented in Table S1. Agreement indices for the initial iteration of this model were $\mathrm{A}_{\text {model }}=60.9$ and $\mathrm{A}_{\text {overall }}=70.2$. In that iteration, three individual dates (Beta-9349, X30285, and Beta251755), had unacceptable individual agreement index values $(A=5.2-56.5)$ and were removed. The resulting sample size of 226 samples produced final model agreement indices of $\mathrm{A}_{\text {model }}=83.7$ and $\mathrm{A}_{\text {overall }}=100.8$.

The Boundary Start age of the amalgamated sample was 363-407 cal AD and the Boundary End was 1072-1146 cal AD The modeled individual date ranges align almost perfectly with these phase boundaries, extending from 365 to $1135 \mathrm{cal} \mathrm{AD}$ at the limits of their respective 95.4\% ranges. As mentioned previously, this estimate of the temporal dimensions of the Middle Period in San Pedro de Atacama should, if anything, serve to overestimate the boundaries of the period, as the sample is known to include some dated events which lie beyond the Middle Period (culturally). If one considers the distribution of individual modeled start and end dates binned by century as in Figure 3, it is clear that the peak intensity of the Middle Period as a "phase" in the San Pedro oases extends from the 7th11 th centuries AD (calibrated), with over $75 \%$ of the individual modeled (start and end) dates falling within that 500-year period.

The most obvious implication of the model at this scale is that the Middle Period in San Pedro appears to have a later onset of peak activity than previously understood. Unsurprisingly, the Middle Period in the oases is specific to the region and only loosely aligned to the larger Andean patterns dubbed the Middle Horizon (e.g., Isbell 2008; Castro et al. 2016). The most recent summation of the region's prehistory (Castro et al. 2016) describes the Middle Period of the oases as having consisted of two phases, Quitor (400-700 AD) and Coyo (700-1000 AD), as based on the earlier stylistic and thermoluminescence works of Tarragó (1968, 1989) and Berenguer et al. (1986). While the overall boundaries of our model are generally in line with this proposition (i.e., our data support the existence of an early Middle Period in San Pedro), the overwhelming majority (more than 75\%) of the individual modeled dates fall in the latter two-thirds of the proposed 600-year span, with some overlap into the early 11 th century AD (calibrated). This concords with data from elsewhere in the Andes that pushes the start of events tied to the subsequent Late Intermediate Period (such as the collapse and partial abandonment of the centers of 
100

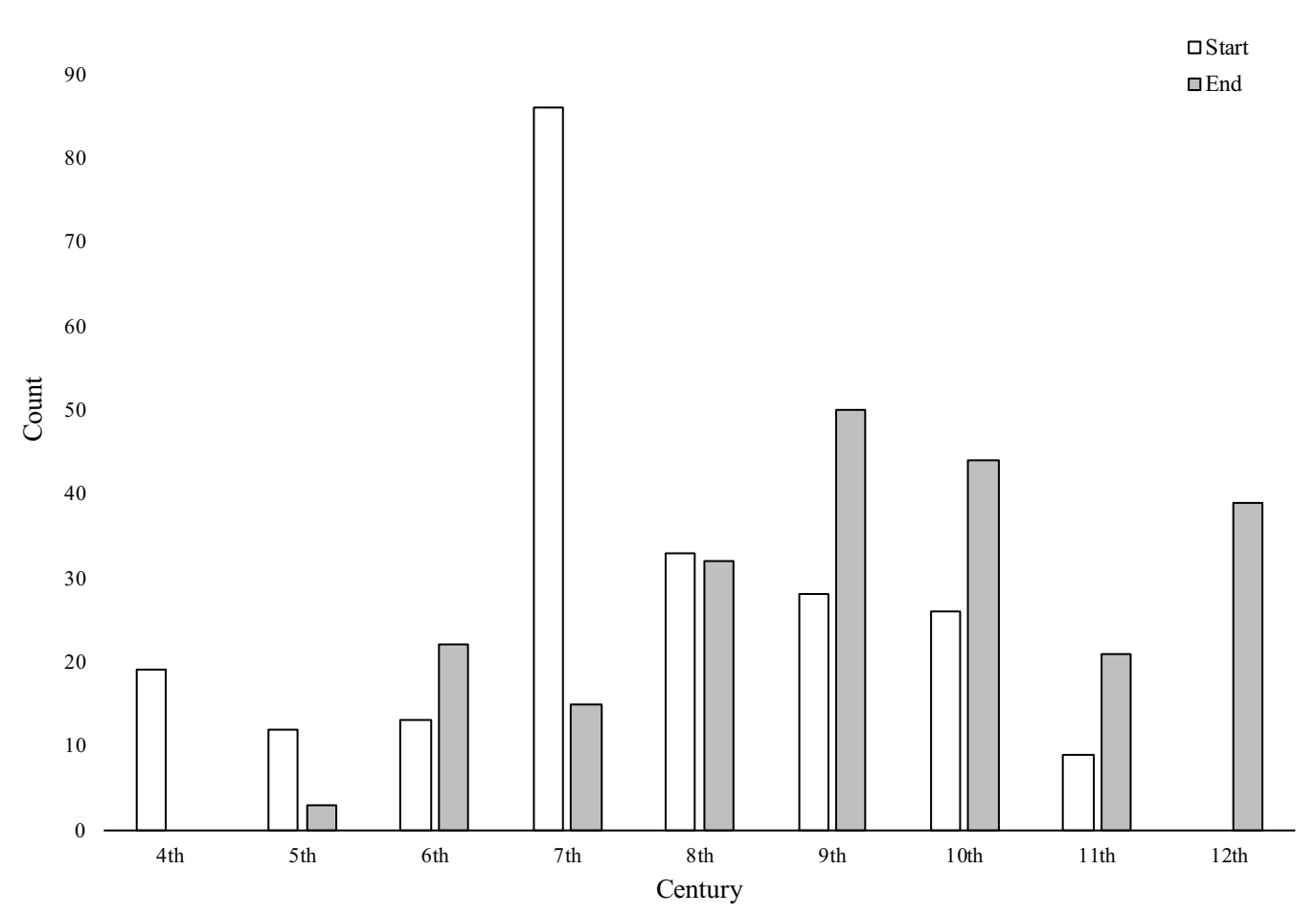

Figure 3 Individual modeled start and end dates binned by century. Note dramatic increase in dates post-600 calAD.

Tiwanaku and Wari) into the 11th century AD (calibrated) (e.g., Janusek 2004; Arkush 2008; Isbell 2008; Jennings et al. 2015). Given our sampling strategy, our findings would appear to support the notion of a florescence of Middle Period culture/society in the later period 600 1000 cal AD. Whether this represents a demographic expansion, or simply greater use of the sampled cemeteries, is irresolvable based on these temporal data alone.

On the basis of this, we support the idea that the chronology of the oases in the Middle Period be refined by employing the evident dichotomy of early (400-600 cal AD) vs. late (600-1000 cal AD), dividing the incipient and established phases of the period without reference to geography. This allows us to step away from the association of specific ayllus with particular phases of occupation. If nothing else, this phasing can be used to structure hypotheses to be tested using other classes of (bio)archaeological evidence.

\section{Inter-Ayllu}

The structure of the ayllu-level modeling is presented in Figure 4, with details of phase boundaries and all individual dates in Table S2. Agreement indices for this iteration were $\mathrm{A}_{\text {model }}=73.6$ and $\mathrm{A}_{\text {overall }}=98.2$. For the sake of comparison, the same model run without outlier trimming produced far lower agreement indices $\left(\mathrm{A}_{\text {model }}=50.1\right.$ and $\left.\mathrm{A}_{\text {overall }}=57.4\right)$, thus validating the initial step taken to mitigate the effects of a small number $(\mathrm{n}=9$ or $3.7 \%$ ) of outlying dated events. Only one individual date (Solcor X30285) had an individual 
OxCal v4.3.2 Bronk Ramsey (2017); r:5 SHCal13 atmospheric curve (Hogg et al 2013)

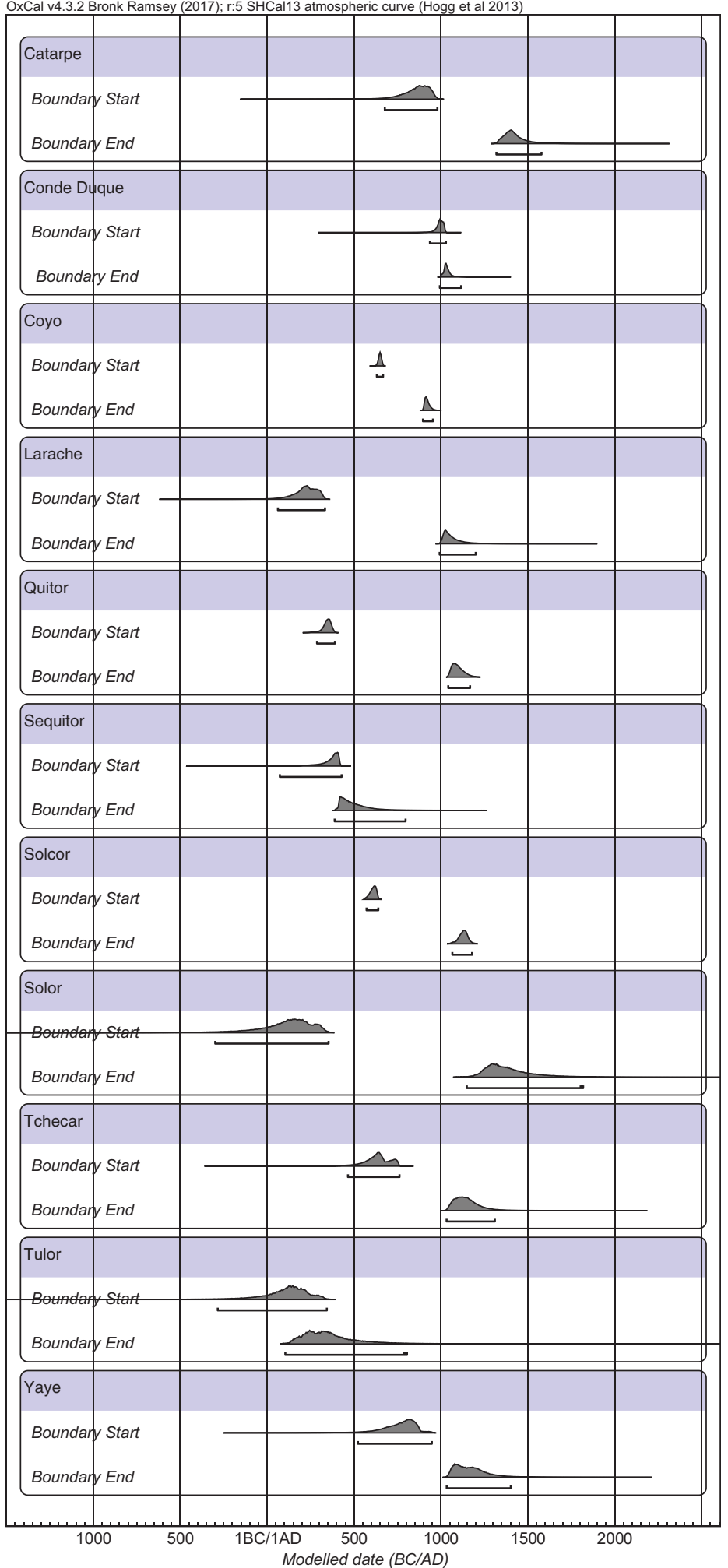

Figure 4 Structure of inter-ayllu model. 
modeled age range with an unacceptable individual agreement index value $(A=22.9)$. With the date removed, the final model agreement indices were $\mathrm{A}_{\text {model }}=110.8$ and $\mathrm{A}_{\text {overall }}=108.4$.

In terms of inception of use (based on the beginning of the modeled "Boundary Start" ages), the earliest-occupied ayllus are the southern Solor and Tulor oases, both of which have Boundary Start ages that begin in the 3rd century BC (calibrated), but with uncertainties that stretch well into the 4th century AD (calibrated). In terms of modeled dates, the range of all four dates from the settlement at Tulor begin in the 1st century BC/AD (calibrated), whereas the earliest Solor individual has a calibrated range beginning in the 2nd century AD (calibrated) and the next earliest date range for that ayllu does not commence until the 4th century AD (calibrated). Tulor thus remains as the ayllu with the earliest evidence of sustained occupation and activity in the San Pedro oases as is supported by archaeological evidence concerning habitation structure and ceramic assemblages (Llagostera et al. 1984; Barón 1986; Llagostera and Costa Junqueira 1999).

Moreover, Tulor and Solor seem to be well-established by the time the next earliest ayllus begin to show evidence of use, as Sequitor and Larache both have Boundary Start date ranges that commence in the 1st century AD (calibrated). In the case of Larache, examination of individual modeled date ranges reveals several samples (4 of 13) with calibrated ranges that begin in the third century AD (calibrated), whereas the limited number (three) of individual determinations from Sequitor all begin in the fourth century AD (calibrated).

Quitor has the next earliest Boundary Start age (288-391 cal AD), which is noteworthy if for no other reason than its span is so much more tightly constrained than any of the other putatively earlier ayllus as a consequence of the much larger number of individual determinations (55) that make up that phase. Both the individual modeled date ranges $(13 / 55,23.6 \%)$ and the Boundary Start age would indicate an earliest occupation of the Quitor ayllu in the fourth century AD (calibrated). Tchecar comes next, with a Boundary Start age that begins in the fifth century AD (calibrated), and with individual modeled ages (three of nine) starting in the 7 th century AD (calibrated).

Both Yaye and Solcor have Boundary Start ages that begin in the 6th century AD (calibrated), but while Solcor has a small number of individual date ranges $(3 / 56,5.4 \%)$ that begin in the 6 th century and $25(44.6 \%)$ that start in the 7th century, the earliest individual age range from Yaye does not begin until the late 7 th century AD (calibrated). On this basis, while Solcor has a somewhat later Boundary Start age than Yaye, we contend that, in the main, the occupation of Solcor predates that of Yaye. This is also corroborated in the material culture of the cemeteries (Torres-Rouff and Costa Junqueira 2006; Castro et al. 2016).

Coyo and Catarpe both present Boundary Start ages in the 7th century AD (calibrated), with the modeled beginning range of Coyo (thirty-six years) being the most constrained of any ayllu presented here. In terms of relative ordering, while $76.6 \%(49 / 64)$ of the individual modeled age ranges from Coyo begin in the 7 th century $\mathrm{AD}$ (calibrated), none of the Catarpe modeled individual ranges begin any earlier than the last decade of the 8th century AD (calibrated). On balance, the occupation of Coyo seems likely to have preceded that of Catarpe. The ayllu with the latest evidence of an onset of activity is Conde Duque, which presents a Boundary Start age and all modeled individual age ranges that begin in the 10th century AD (calibrated). It should be noted that the sample for Conde Duque is small and all derived from one cemetery. Moreover, the center of the modern town of San Pedro sits atop much of this ayllu, thus obscuring more detailed insights into its past. 
Turning to the cessation of activity by ayllu (as judged by the end of the various Boundary End ranges), Sequitor and Tulor are the two localities with the earliest termination of activity. Occupation of Sequitor has the earliest end to its Boundary End age (389-797 cal AD), but with individual modeled age ranges that end in the 5th to 6th centuries AD (calibrated). Tulor possesses only a slightly later end to its Boundary End age (105-805 cal AD) but has individual modeled age ranges that are far more restricted, with ranges that end between the 4th and 6th centuries AD (calibrated). With the limited number of available dates from these ayllus, it is difficult to determine the relative sequence of activity cessation, although occupation of Tulor may have ended slightly earlier.

The Boundary End age (897-955 cal AD) of Coyo comes next, suggesting a limited (250-300 year) overall duration of occupation and use of that ayllu, when one considers that the onset of activity there falls in the mid-7th century AD (calibrated). It is noteworthy that the entirety of the Boundary End range for Coyo is exclusive of any other ayllu's Boundary End, suggesting that the cessation of activity at Coyo was wholly temporally distinct from the end of the activity at any other ayllu. Moreover, none of the individual modeled age ranges extend until even the middle of the $10^{\text {th }}$ century AD (calibrated). This finding is in line with earlier observations that the Coyo ayllu, for example, shows distinct patterns of trauma (Torres-Rouff et al. 2018) and the presence of exotic tropical diseases (Costa Junqueira et al. 2009; Marsteller et al. 2011; Costa Junqueira and Llagostera 2014), as compared with many of its neighbors.

Activity at Conde Duque would appear to have ended next, sometime in the late 10th-early 12th century AD (calibrated). The modeled ranges for all seven of the samples from Conde Duque fall entirely within the 10th/11th centuries AD (calibrated), indicating a short and intense period of activity, as was the case with Coyo. Quitor emerges as the ayllu with the next Boundary End age range termination, falling between 1043-1168 cal AD. There is a substantial number of samples $(12 / 55,21.8 \%)$ with modeled ranges that terminate in the 11 th or 12th century AD (calibrated), suggesting that its use life/occupation persisted until, or beyond, that of Conde Duque.

Both Solcor and Larache have Boundary End date ranges that terminate in the late 12th/early 13th century AD (calibrated). The range of Larache's Boundary End (993-1201 cal AD) begins earlier and ends later than that of Solcor (1066-1180 cal AD). Comparing individual dates, only three samples from Larache have modeled age ranges that extend into the first half of the 11th century AD (calibrated), while there are 16 samples $(29.1 \%)$ from Solcor with modeled age ranges extending into the 12th century AD (calibrated). On this basis, the cessation of activity at Larache would appear to predate that seen at Solcor.

The beginnings of the Boundary End dates ranges for the next two ayllus, Tchecar (1033-1311 cal AD) and Yaye (1034-1402 cal AD), are essentially contemporary, although the end of the Yaye range is nearly a full century later than that of Tchecar. There are, however, fairly small sample sizes representing each ayllu, making detailed inferences about their relative ordering rather speculative.

Finally, the ayllus of Catarpe and Solor present the two latest-concluding Boundary End ranges, 1320-1579 cal $\mathrm{AD}$ and 1149-1819 cal AD, respectively. The latest individual modeled age for Catarpe extends only to the early 15th century AD (calibrated), while at Solor the latest individual age range ends in the late 14th century AD (calibrated). It is the small sample size and wide variance of the individual dates from Solor that pushes the Boundary End range into the 19th century AD (calibrated), much later than that seen for 


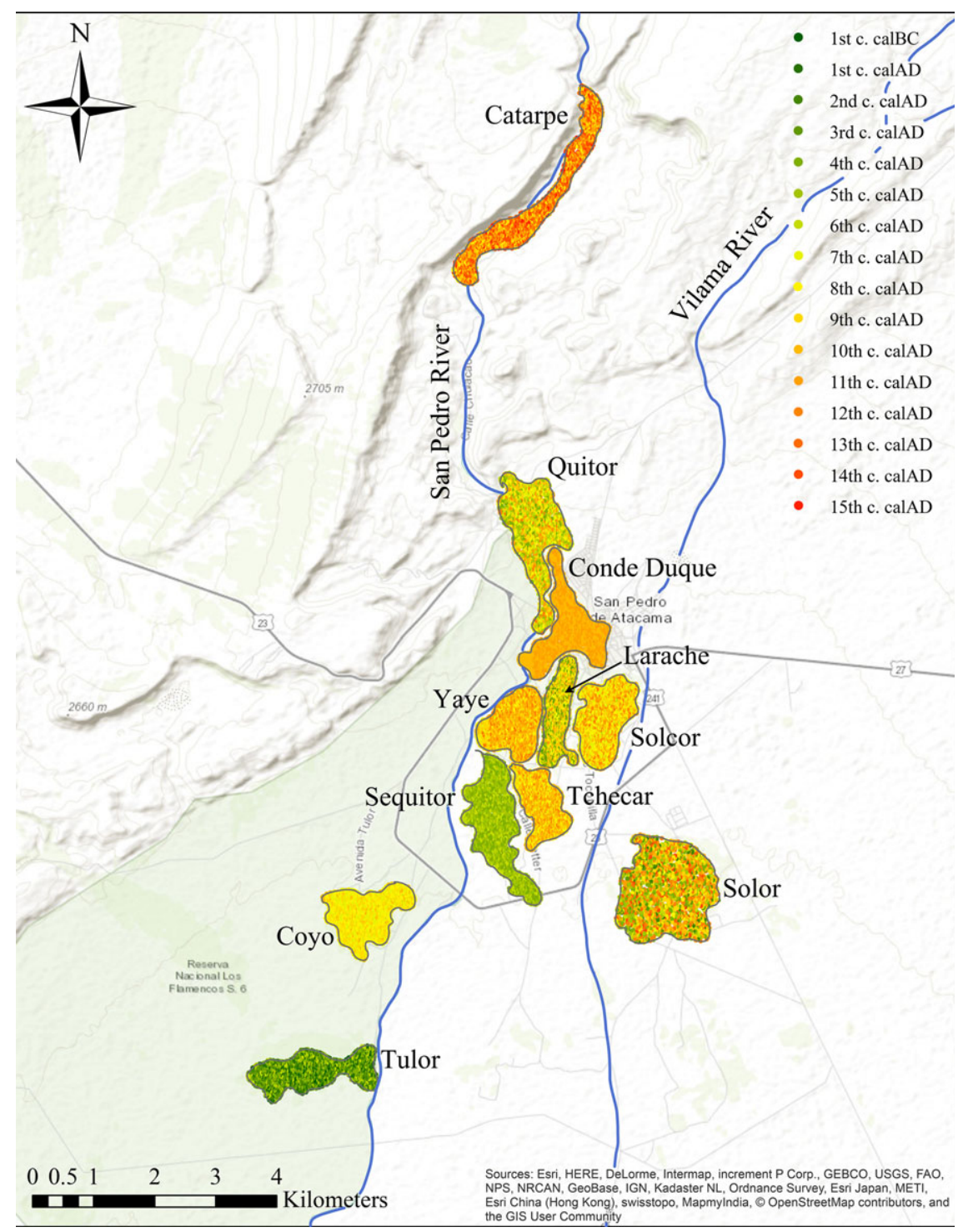

Figure 5 Heat map of inter-ayllu dates by century.

Catarpe. One imagines that a larger sample size for Solor might bring its modeled conclusion into greater alignment with that seen for Catarpe.

In summary, our results indicate that the sequence of initial use of the various tested ayllus is as follows: Tulor, Solor, Larache, Sequitor, Quitor, Tchecar, Solcor, Yaye, Coyo, Catarpe, and Conde Duque. The sequence of the cessation of use of the various tested ayllus is as follows: Tulor or Sequitor, Coyo, Conde Duque, Quitor, Larache, Solcor, Tchecar or Yaye, and finally Catarpe or Solor. These results are visualized, in a summary form in Figure 5. 
Taken together, the chronological modeling of cemetery use across the different ayllus reveals a complex pattern of human occupation. The chronological pattern of occupation of the oases has been of particular interest to archaeologists (Orellana 1964; Llagostera and Costa Junqueira 1999; Uribe 2002; Núñez et al. 2010; Agüero and Uribe 2011; Torres-Rouff and Hubbe 2013; Uribe et al. 2016), due to their geographic and ecological circumscription, and to the role that said circumscription may have played in shaping the human presence therein. Indeed, the perceived challenge of occupying the limited fertile land in the oases has driven much of the discussion about the human occupation in the area. Previous studies have suggested a general south-to-north succession of human settlements over the oases, with access to, and control over, water playing an important role in population displacement across the oases over time (Llagostera and Costa Junqueira 1999; TorresRouff and Hubbe 2013). Our results speak directly to this discussion and depict a pattern of occupation that is considerably more nuanced than previously considered.

The refined results obtained with the chronological modelling of ayllu/cemetery use reveal considerable variation in the pattern and length of occupation of the oases. While the earliest ayllus to be occupied are in the southern portion of oases (Tulor and Solor) and the last to show human presence is the northernmost ayllu of Catarpe, the rest of the ayllus do not show any directional pattern of occupation. For instance, the northern ayllu of Quitor has earlier Boundary Start dates than most of the central and south located ayllus. As such, the reasoning that led to the human expansion across the oases during the Middle Period cannot be easily ascribed to a solely geographic logic.

On the other hand, our results support previous suggestions that access to water sources played a role in the length of occupation of individual ayllus. Tulor shows a short modeled period of occupation when compared to Solor, which shows the longest range of any ayllu in the oases. This pattern has been noted before (Torres-Rouff and Hubbe 2013) and has been suggested to be the result of past changes in river courses. Tulor and the other western ayllus (Coyo and Yaye) are located close the San Pedro river, which shifted course significantly in the past (Llagostera et al. 1984; Niemeyer 1989). These three ayllus show relatively short periods of occupation, which supports the hypothesis of a changing path of the San Pedro River. Solor, on the other hand, is directly associated with the Vilama River, which has a narrower riverbed near this ayllu and only shows evidence of having shifted farther south of the ayllu. As such, it would have sustained the longer period of occupation in the latter ayllu.

Finally, it is noteworthy that the pattern of occupation of the oases during the period studied is not tied to region-wide events, since the times of occupation and abandonment of the studied cemeteries show no common chronological dimensions. As such, these results demonstrate that human expansion in the oases largely responded to factors intrinsic to each of the ayllus, reflecting micro-environmental differences between them, local socio-cultural dynamics, or other local factors. As such, the chronological diversity of cemetery use across ayllus suggests local independence in defining the period and duration of cemetery use, complementing the archaeological and bioarchaeological literature that demonstrates significant differences and inequalities between ayllus during the Middle Period (e.g., Torres-Rouff 2011). 
OxCal v4.3.2 Bronk Ramsey (2017); r:5 SHCal13 atmospheric curve (Hogg et al 2013)

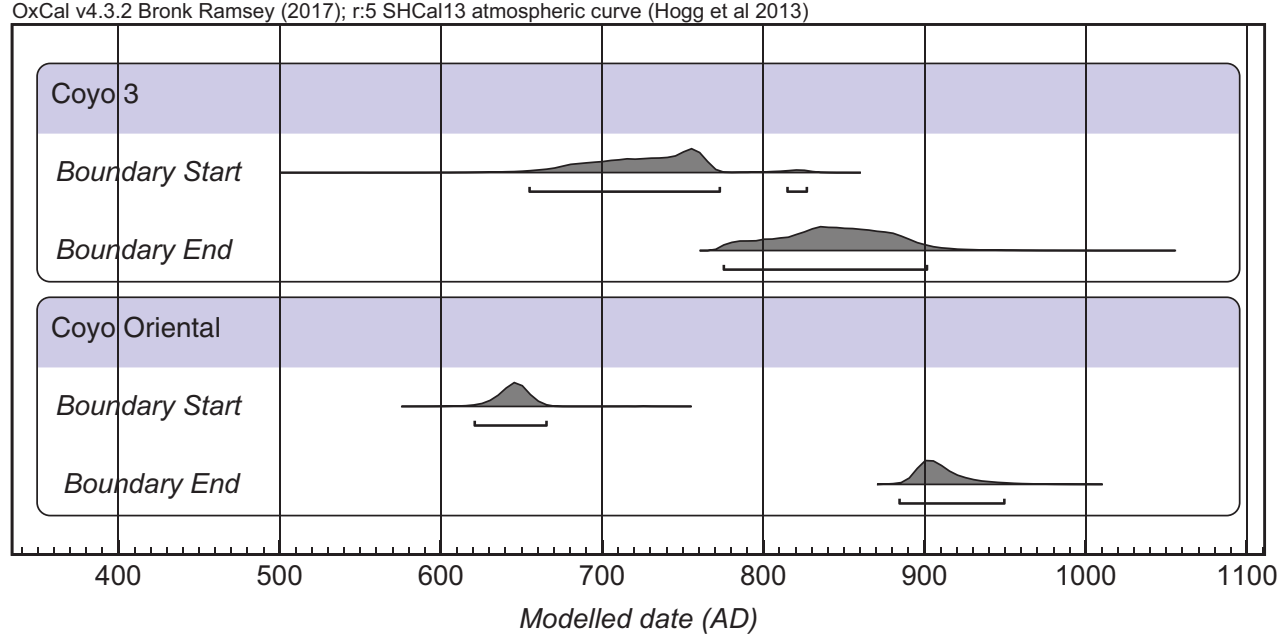

Figure 6 Structure of intra-ayllu model for Coyo.

\section{Intra-Ayllu}

As noted previously, the more in-depth sampling approach taken to sites in the Coyo, Quitor, and Solcor ayllus has resulted in an abundance of dates for some of the ten cemeteries in those three ayllus: Coyo Oriental and Coyo 3, Quitor 1, 2, 5, 6, and 8/9, and Solcor 3, Solcor Plaza, and Solcor Nueva Población. Below we explore these in more detail, assessing both sequencing and duration of use for each ayllu and cemetery.

\section{Coyo}

The structure of the model for the two Coyo cemeteries is presented in Figure 6, with details of phase boundaries and all individual dates in Table S3. Agreement indices for this model were $\mathrm{A}_{\text {model }}=84.8$ and $\mathrm{A}_{\text {overall }}=78.4$. Two dates from Coyo 3 (X30221 and AA111819) had agreement index values below the suggested index cutoff value of $60.0(\mathrm{~A}=50.4$ and 48.8 , respectively). Removal of these two dates increased the model's agreement indices to $\mathrm{A}_{\text {model }}=97.1$ and $\mathrm{A}_{\text {overall }}=94.7$.

The model for Coyo indicates complete overlap, and even contemporaneity, of the two cemeteries considered here. The Boundary Start age for Coyo Oriental (621-665 cal AD) slightly predates that of Coyo 3 (655-827 cal AD), while the Boundary End date of Coyo Oriental (884-949 cal AD) is slightly later but largely overlapping with that of Coyo 3 (775-901 cal AD). If any difference can be detected on the basis of the ranges of individual modeled dates, it would appear that use of Coyo Oriental both commences slightly earlier and ends slightly later than that of Coyo 3, with differences at both extremes of forty to sixty years.

In terms of the duration of use of the Coyo cemeteries, the use of Boundary Start and End ages indicates that Coyo 3 was in use for approximately 250 years, while Coyo Oriental had some 220-330 years of use. The several century use-life of these cemeteries is quite different to that observed in Quitor but is in line with what was observed for Solcor. 
Regardless of these differences, the Coyo cemeteries would appear to have been used largely contemporaneously for some two to three centuries despite their shared location in the Coyo ayllu. The cemeteries themselves are geographically distinct (Figure 1), occupying the southwest and northeast corners of the small area of Coyo. Given that time does not distinguish the cemeteries, the concurrent use of these two separate cemetery precincts may represent social distinctions internal to that ayllu. The established distinctions in mortuary goods, for example in so-called luxury items such as metals and foreign objects, between these cemeteries may in fact reflect some form of intra-ayllu differentiation tied to social status (Oakland 1992; Costa Junqueira and Llagostera 1994; Cocilovo et al. 2011; TorresRouff et al. 2018).

\section{Quitor}

The structure of the model of the five Quitor cemeteries is presented in Figure 7, with details of phase boundaries and all individual dates offered in Table S4. Due to complete temporal overlap, all dates from Quitor 6 (both the putatively earlier section and that denoted by excavators as Quitor 6 Tardío [late]) were combined and treated as one cemetery, as were samples from cemeteries Quitor 8 and 9, which were combined on the basis of their geographic proximity (being separated only by a modern road). The resulting five groupings used in the model were Quitor 1, 2, 5, 6, and 8/9. Agreement indices for this model were $A_{\text {model }}=98.4$ and $A_{\text {overall }}=96.3$. No individual dates had agreement index values below the stipulated cutoff of 60.0 . It should be noted that there are large differences in sample size among these different cemeteries, with Quitor 1 and 2 being represented by only one and five dates, respectively, while the other three cemeteries have sample sizes of 10 to 22 dates each. Direct comparisons of use-lives of these cemeteries must thus be viewed as being somewhat contingent.

As at Coyo, the modeled use-life of the cemeteries of Quitor reveals a large degree of simultaneous cemetery use. On the basis of both Boundary Start ages and individual modeled age ranges, Quitor 2 and 5 were in-use from the mid-3rd century AD (calibrated), with the use of Quitor 8/9 commencing at the same time or soon thereafter (depending on whether one considers the Boundary Start or individual modeled age range). By the late 5th century AD (calibrated), Quitor 6 begins to be used as well, such that by the 5 th/6th century AD (calibrated), there are at least four cemeteries in simultaneous use within the Quitor ayllu. This number rises to five cemeteries if we take the one available date from Quitor 1 (X14968A, 1030-1210 cal AD) to be representative of sustained activity there. Unlike the case of Coyo, several of the Quitor cemeteries are in close geographic proximity, and therefore it is unlikely that they represent clear spatial distinctions in use.

The collective activity in these cemeteries begins to wane in or after the 8th century AD (calibrated), as Quitor 5 (Boundary End age 812-1048 cal AD), Quitor 6 (Boundary End age 1026-1145 cal AD), Quitor 8/9 (Boundary End age 1013-1378 cal AD), and Quitor 2 (Boundary End age 700-1381 cal AD) all cease evidence of use between the 8 th and 14th centuries AD (calibrated).

The modeled duration of the three Quitor cemeteries for which we possess sufficient sample size to speak with confidence reveals a pattern very distinct from that observed for Coyo. As based on Boundary Start and End ranges, Quitor 5 was used for some 430-840 years, Quitor 6 for 400-730 years, and Quitor 8/9 for 500-1190 years. Even at their lower limits, these use-life 


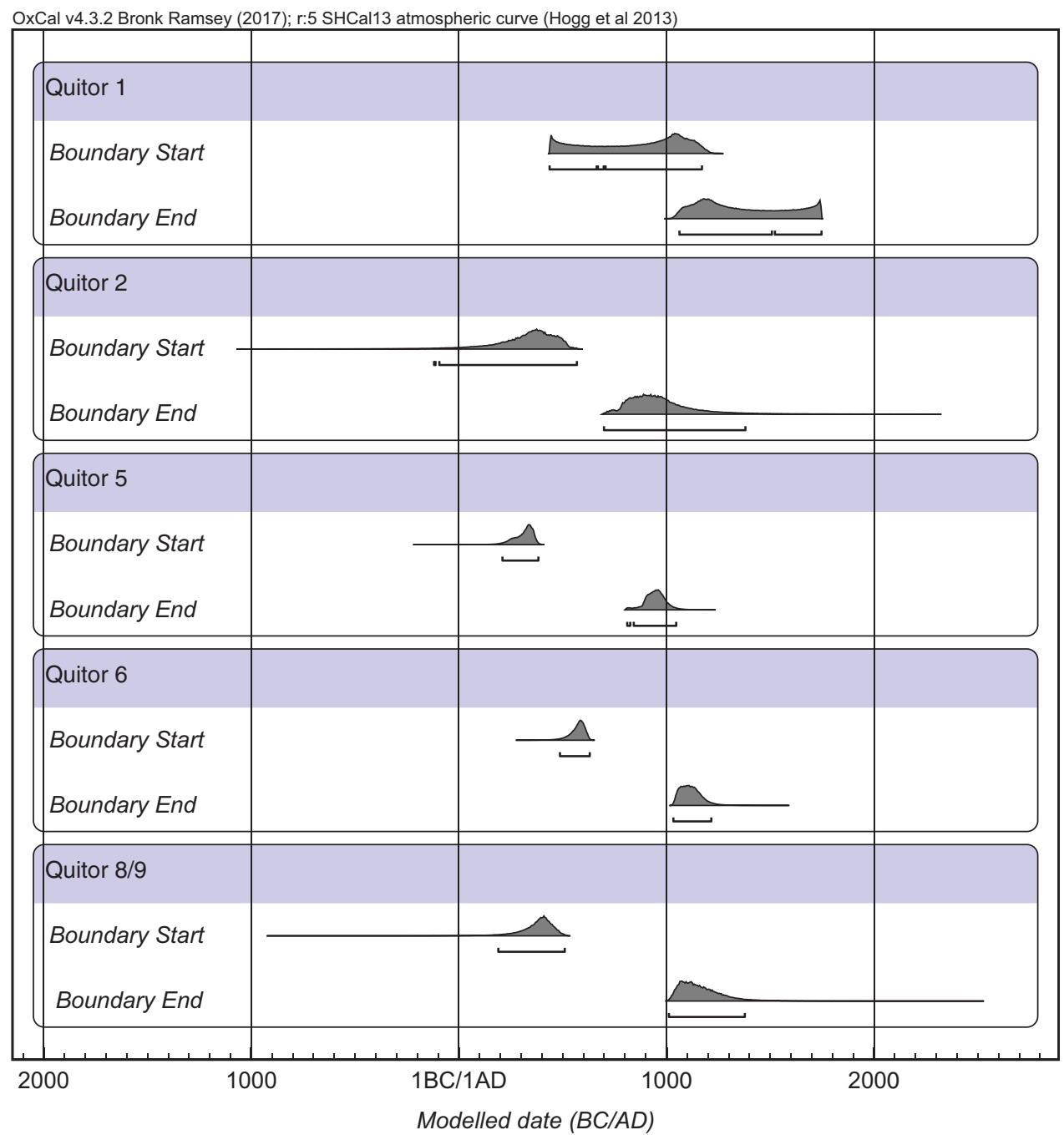

Figure 7 Structure of intra-ayllu model for Quitor.

periods are appreciably longer than those of the Coyo or Solcor cemeteries. That said, the data also suggest the clear contemporaneity of multiple cemeteries like seen at Coyo, which may reflect their use by lineage groups or other forms of social groupings that had salience over a long period of time.

\section{Solcor}

The structure of the model of the three Solcor cemeteries is presented in Figure 8, with details of phase boundaries and all individual dates offered in Table S5. Agreement indices for this model were $A_{\text {model }}=97.1$ and $A_{\text {overall }}=93.4$. No individual dates had agreement index values below the stipulated cutoff of 60.0 . 


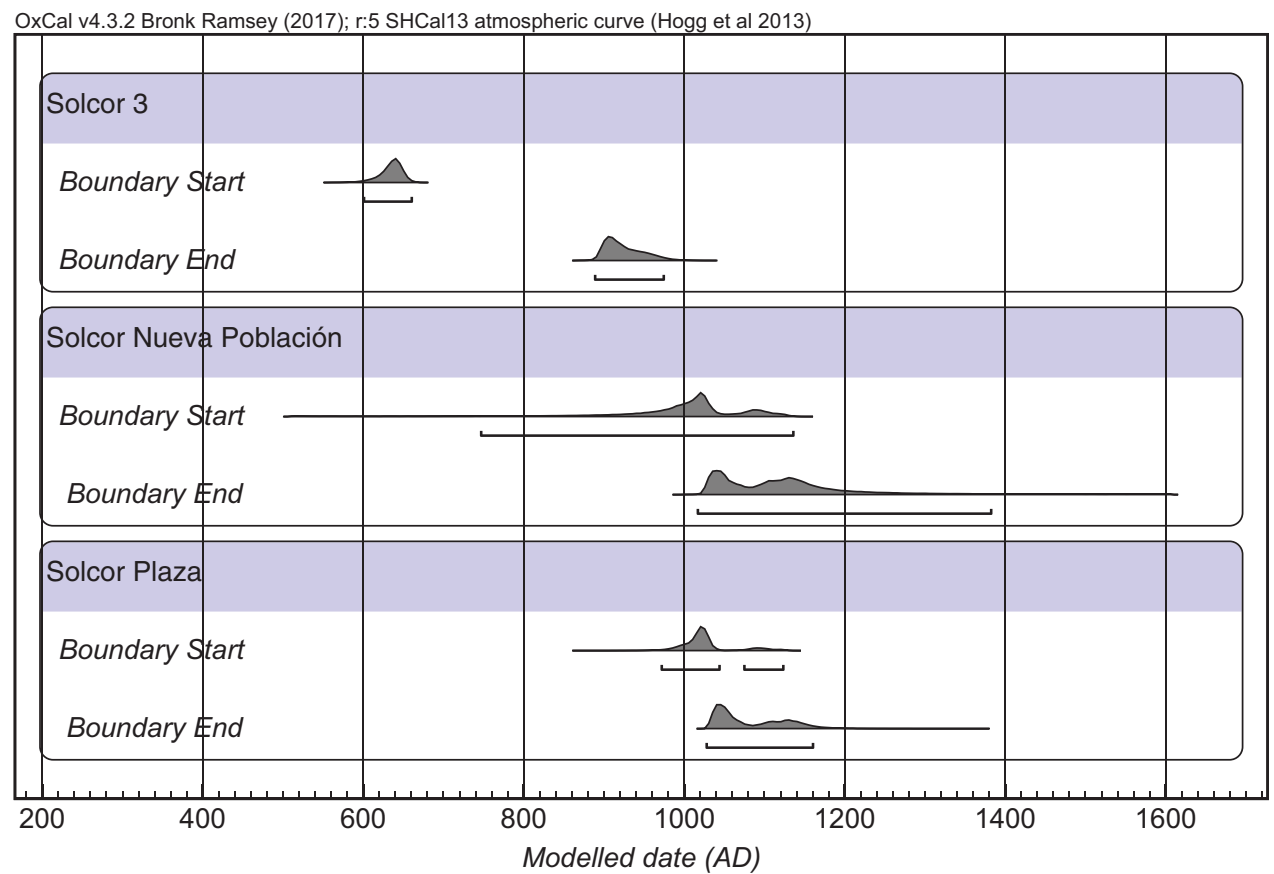

Figure 8 Structure of intra-ayllu model for Solcor.

Unlike Coyo or Quitor, at Solcor, the intra-ayllu model revealed a clear sequence, and even succession, of cemetery use. Solcor 3 has the earliest onset of use, with a Boundary Start age range of 601-660 cal AD, and with the earliest individual modeled age ranges commencing in the first two decades of the 7th century AD (calibrated). It is not until nearly the conclusion of Solcor 3's Boundary End range (889-974 cal AD) that activity begins at Solcor Plaza (Boundary Start age 972-1123 cal AD) or, as based on individual modeled age ranges, at Solcor Nueva Población, where the earliest samples' ranges do not commence until the last decade of the 10th century AD (calibrated). While the small sample size for Solcor Nueva Población $(n=3)$ precludes any categorical statement about use-life, there is clear evidence at Solcor 3 and Solcor Plaza of a succession of cemetery use, with activity at Solcor Plaza commencing at, or within a generation of, the cessation of activity at Solcor 3. Ultimately, activity at both of the later cemeteries ceases by, most likely, the 12th century AD (calibrated) (based on the Boundary End age at Solcor Plaza and the end of the individual modeled age ranges at Solcor Nueva Población).

The duration of use of the larger Solcor cemeteries shows greater similarity to the pattern observed at Coyo rather than that documented for Quitor. The results of the modeled Boundary Start and End ranges indicate that Solcor 3 was in use for 230-370 years, while Solcor Plaza was utilized for up to 190 years. In contrast to the two other ayllus we have studied more closely, the temporal data provides no clear evidence to support the idea that existing social structures limited access to cemetery use at Solcor, as this ayllu's cemeteries show limited concurrent use. 


\section{DISCUSSION AND CONCLUSION}

The three-tiered modeling of these 243 dates from the cemeteries of San Pedro de Atacama has yielded a series of novel insights into the chronological structure of Middle Period life and cemetery use in the oases. Furthermore, the results generated allow us to formulate a series of questions, and even explicit hypotheses, that will be examined and tested in our future analysis of a suite of archaeological, bioarchaeological, and isotopic data from the individuals and cemeteries considered here. As this work is currently in progress, we cannot yet speak to the impact of these results on our broader bioarchaeological endeavor. Instead, we consider below only a few of these new insights and focus more extensively on directions for future testing on the all ayllus, inter-ayllu, and intra-ayllu scales. Finally, we conclude by offering more general thoughts about the implications of these results for our understanding of lifeways in San Pedro de Atacama's Middle Period and for future archaeological and bioarchaeological work conducted therein.

At the coarsest spatial scale (all ayllus), we call attention to the evident dichotomy of early/ incipient (400-600 cal AD) vs. late/established (600-1000 cal AD) phases of the Middle Period in the San Pedro de Atacama oases. While this dichotomy is clear in the assembled radiometric corpus, we present this phasing not as novel dogma, but rather as a crucial aspect of the oases' occupation that should be examined in-and tested by-other archaeological work in the region moving forward. Crucially, this proposed division can be used to generate a series of structuring questions by which future work could be guided, for instance by examining whether various embodied social phenomena (inequality, body use, diet), or indeed the stylistic dimensions of a myriad of material culture, change between these proposed phases. Indeed, previous studies already have identified shifts in the production of certain ceramic styles (Stovel 2013, the disappearance of the Rojo Pulido style) or the diminution of San Pedro as a ceramic production center (Gallardo et al. 2017) in the century between 600-700 cal AD. The robustness of this possible moment of transition deserves examination across a broader range of artifacts and archaeological data. Moreover, in light of the discussions surrounding the mediated form that Tiwanaku influence took in the oases, it is possible that this moment of disjuncture around $600 \mathrm{cal}$ $\mathrm{AD}$ represents an inflection point in the intensity, level, or mechanism of that highland polity's presence in San Pedro, a phenomenon that could, conceivably, be visible in some classes of (bio)archaeological data.

Moving to the inter-ayllu scale, the results presented here shed new light on long-running debates about purported similarities and differences between and among ayllus and the drivers of suggested diachronic shifts in their use and occupation. In broad terms, our data show that some of these previous ad hoc models have been overly simplistic, while these new formulations reveal (or support) far greater complexity. As seen in Figure 5, notions of an overall south-to-north succession of occupation in the oases find little support, whereas closer proximity to water engendered long term occupation as compared to those communities where water became scarce over time due to movement of river channels (for example, at Tulor, Coyo, or Yaye) and occupation/cemetery-use were short-lived (a finding that supports previous suggestions in Torres-Rouff and Hubbe 2013).

Most crucially, this model iteration stresses the independence of the trajectories of different ayllus, with local conditions being the best predictor of chronological patterns of cemetery use. Patterns of occupation of the oases during the Middle Period are not simply echoes of region-wide events as the dynamics of human activity in each ayllu responded to local 
environmental or socio-cultural dynamics. This finding buttresses the archaeological and bioarchaeological literature showing significant differences and inequalities between ayllus during the Middle Period. For example, our previous research has documented radical differences in the presence of violent injury between individuals interred at different cemeteries in this period (Torres-Rouff 2011; Torres-Rouff et al. 2018), while, simultaneously, other aspects of mortuary treatment (e.g., the provisioning of Negro Pulido ceramics) are standardized to the point of ubiquity across time and ayllus (Stovel 2005).

Clearly, with the temporal framework we provide here in hand, much more work on the nuances of inter-ayllu differences is merited, further exploring the processes described by Salazar and colleagues who noted that, "during the [Middle Horizon] the local community of [San Pedro de Atacama] created and reproduced social boundaries and affiliations at different levels simultaneously" (Salazar et al. 2014: 148). Indeed, Salazar and colleagues identified inter-ayllu variation in cranial modification, the presence and ubiquity of paraphernalia associated with the use of hallucinogenic snuffs, and metallurgical practices, among other practical and material differences, which deserve re-examination with the added context of the temporal modeling presented here.

Finally, just as these results reveal diversity between/among ayllus, at the finest scale, they also indicate differences in intra-ayllu patterns of cemetery use. In sum, what these analyses expose is that intra-ayllu patterns of cemetery use in San Pedro de Atacama's Middle Period were not homogenous and, consequently, are likely to reflect a society in which there existed multiple distinct mortuary practices (in terms of the designation and maintenance of cemetery precincts/ burial space). The three ayllus we examine in greater detail here reveal three distinct patterns of use, suggestive of a lack of standardization of the use of mortuary spaces across the ayllus. While some burial spaces were used for centuries, others had more discrete and constrained use-lives. Similarly, some small communities had several cemeteries in use at a given time (which may reflect their use by lineage groups or other forms of social groupings that had salience over a long period of time), while others had a sole burial location. Thus, while the identity of ayllus is, at least in part, chronological, the multiplicity of patterns ultimately suggests a complex picture of cemetery use in the Middle Period that cannot be parsed with temporal data alone and calls for the integration of bioarchaeological and archaeological data.

The full meaning or motivation behind these distinct patterns awaits consideration of other classes of (bio)archaeological data, and the social differences reflected in cemetery use are a crucial future distinction for analyses. However, based on these observed differences, we might expect differences in artifact style, mortuary treatment, and lived experience (as evidenced by bioarcheology) to vary in distinct ways among the cemeteries of the different ayllus considered here. Crucially, these data allow us to disaggregate the effects of diachronic change from synchronic variation, permitting far more nuanced comparisons of difference observed among the mortuary goods of Coyo or Quitor, where multiple cemeteries were in use at the same time, and Solcor, where there is a chronological distinction between periods of use of different mortuary spaces. Specialists in material culture will be able to explore these possibilities in varied materialities. Regardless of the outcomes of these future studies, what is happening among and within the San Pedro ayllus is notably distinct, and, even after nearly a century of study, merits more detailed consideration. 
At its broadest, this study permits the formulation of new questions, which can be examined in future studies and provoke the re-analysis of decades of accumulated archaeological work in the San Pedro oases. While we cannot say with certainty what the impact of the incorporation of this understanding of these results will be for our understanding of life in San Pedro in the Middle Period, they could well provide ample ground for radical transformations thereof. At the very least, these findings lay bare the fact that, just as there was no one Middle Period, let alone Middle Horizon, experienced across the southern Andes for a 600-year period, there likely was not a homogenous experience of the Middle Period in San Pedro de Atacama over time, across, or within ayllus. We then raise a series of possibilities. Does 600 cal AD represent a meaningful moment of disjuncture across different classes of evidence? What might detailed archaeological study of textiles, ceramics, snuff paraphernalia and other material culture reflect when tied to the distinctions between cemeteries and time? Do the divergent trajectories of cemetery use and occupation seen among and within ayllus represent not just distinct responses to local environmental conditions, but also different strategies/levels/intensities of interaction with the Tiwanaku polity? Indeed, these results, rooted in the modeling of such a large corpus of radiocarbon assays, may form the basis for a re-thinking of long-accepted social development models in the region.

Ultimately, it is clear that these results can help to reduce the equifinality of different proposed explanations of social change, if for no other reasons than they eliminate the confounding effect of an inability to differentiate between synchronic variation and diachronic change. Indeed, we hope the structure this analysis provides will serve as a useful scaffolding for a host of future detailed examinations of stylistic variation, settlement patterning, mortuary analysis, and bioarchaeology in the San Pedro oases in the coming decades.

\section{ACKNOWLEDGMENTS}

Portions of this research were supported by the National Science Foundation, Grants BCS1358753 and BCS-1359644 and the Vicerrectoría de Investigación y Desarrollo Tecnológico of the Universidad Católica del Norte. This work would not have been possible without the continued support of the staff of the Instituto de Arqueología y Antropología and the Museo Arqueológico Le Paige of the Universidad Católica del Norte, in particular, M. Arturo Torres and Jimena Cruz. We thank Mariana Ugarte for the design and execution of Figure 1. Finally, the authors wish to acknowledge the efforts of our colleague Sarah Schrader as well as our thoughtful and hard-working student research assistants: Cameron Beason, Brianne Herrera, Rocío López-Barrales, Eva Mann, Evan Mann, Camila MoralesZuñiga, and Erin K. Smith.

\section{SUPPLEMENTARY MATERIAL}

To view supplementary material for this article, please visit https://doi.org/10.1017/RDC. 2020.105

\section{REFERENCES}

Abercrombie TA. 1998. Pathways of memory and power: ethnography and history among an Andean people. Madison (WI): University of Wisconsin.
Agüero C. 2004. Componente Tiwanaku vs. componente local en los oasis de San Pedro de Atacama. In: Solanilla V, editor. Textiles Andinos: pasado, presente y futuro. Barcelona: 
Universidad Autónoma de Barcelona. p. 180-198.

Agüero C, Uribe M. 2011. Las sociedades formativas de San Pedro de Atacama: asentamiento, cronología y proceso. Estudios Atacameños 42:53-78.

Agüero C, Uribe M 2014. Rethinking the Tiwanaku phenomenon in San Pedro de Atacama through the study of the textiles of Solcor 3 and their associated contexts (400-1000 AD). Textile Society of America Symposium 2014 Proceedings. Los Angeles.

Arkush E. 2008. War, chronology, and causality in the Titicaca Basin. Latin American Antiquity 19(4):339-374.

Barón AM. 1986. Tulor: posibilidades y limitaciones de un ecosistema. Chungara 16-17:149-158.

Berenguer J. 1998. La iconografía del poder en Tiwanaku y su rol en la integración de zonas de frontera: una hipótesis. Boletín del Museo Chileno de Arte Precolombino 7:19-38.

Berenguer J, Román A, Deza A, Llagostera A. 1988. Testing a cultural sequence for the Atacama Desert. Current Anthropology 29:341-346.

Berenguer J, Deza A, Román A, Llagostera A. 1986. La secuencia de Myriam Tarragó para San Pedro de Atacama: un test por termoluminiscencia. Revista Chilena de Antropologia 5:17-54

Bronk Ramsey C. 1995. Radiocarbon calibration and analysis of stratigraphy: the OxCal program. Radiocarbon 37(2):425-430.

Bronk Ramsey C. 2009. Bayesian analysis of radiocarbon dates. Radiocarbon 51(1):337-360.

Browman DL. 1980. Towards the development of the Tiahuanaco (Tiwanaku) state. In: Browman DL, editor. Advances in Andean archaeology. p. 327349. The Hague: Mouton.

Browman DL. 1997. Political institutional factors contributing to the integration of the Tiwanaku state. In: Manzanilla L, editor. Emergence and change in early urban societies. p. 229-243. New York: Plenum Press.

Campbell R. 2015. Nota historiográfica sobre las transcripciones del "Congreso Internacional de Arqueología de San Pedro de Atacama" y la creación de la Sociedad Chilena de Arqueología. Serie Monográfica de la Sociedad Chilena de Arqueología 5:1-25.

Castro V, Berenguer J, Gallardo F, Llagostera A, Salazar D. 2016. Vertiente Occidental Circumpuñena. Desde las sociedades posarcaicas hasta las preincas (ca. 1.500 años a.C. a 1.470 años d.C.). In: Hidalgo J, Schiappacasse V, Niemeyer H, Aldunate C, Solimano I, editors. Culturas de Chile: Prehistoria Desde sus Orígenes Hasta los Albores de la Conquista. Santiago de Chile: Editorial Universitaria. p. 239-283.

Cock G. 1981. El ayllu en la sociedad Andina: alcances y perspectivas. In: Castelli A, de Paredes MK, de Pease MM, editors.
Etnohistoria y Antropología Andina. p. 231253. 2nd ed. Lima: Jornada del Museo Nacional de Historia.

Costa Junqueira MA. 1988. Reconstitución física y cultural de la población tardía del cementerio de Quitor-6 (San Pedro de Atacama). Estudios Atacameños 9:99-126.

Costa Junqueira MA, Llagostera A. 1994. Coyo-3: momentos finales del periodo medio en San Pedro de Atacama. Estudios Atacameños 11:73-143.

Costa Junqueira MA, Llagostera A. 2014. Leishmaniasis en Coyo Oriente: migrantes trasandinos en San Pedro de Atacama. Estudios Atacameños 47:5-18.

Costa Junqueira MA, Matheson C, Iachetta L, Llagostera A, Appenzeller O. 2009. Ancient leishmaniasis in a highland desert of northern Chile. PLoS One 4(9).

Cocilovo JA, Llagostera A, Varela HH. 2011. Armando el Rompecabezas en San Pedro de Atacama: El Sitio Coyo Oriental y la Cuestion de los Sectores del Padre Le Paige desde la Antropología Biológica. Revista de Antropología 23(1):149-172.

Figueroa V, Salazar D, Salinas H, Núñez-Regueiro P, Manríquez G. 2013. Pre-Hispanic mining ergology of northern Chile: an archaeological perspective. Chungara Revista de Antropología Chilena 45:61-81.

Gallardo F, Correa I, Pimentel G, Blanco JF. 2017. Consumption consumes: circulation, exchange, and value of San Pedro de Atacama black polished ceramics. Latin American Antiquity 28(2):252-268.

Goldstein PS. 2000. Communities without borders. The vertical archipelago and diaspora communities in the southern Andes. In: Canuto MA, Yaeger J, editors. Archaeology of communities: a New World perspective. Taylor and Francis Group. p. 182-209.

Hogg AG, Hua Q, Blackwell PG, Niu M, Buck CE, Guilderson TP, Heaton TJ, Palmer JG, Reimer PJ, Reimer RW, Turney CS. 2013. SHCal13 Southern Hemisphere calibration, 0-50,000 years cal BP. Radiocarbon 55(4):1889-1903.

Hubbe M, Oviedo M, Torres-Rouff C. 2011. El estado de conservación de la colección osteológica Gustavo Le Paige y su contextualización cronológica. Estudios Atacameños 41:29-44.

Hubbe M, Torres-Rouff C, Neves WA, King LM, Da-Gloria P, Costa MA. 2012. Dental health in Northern Chile's Atacama oases: evaluating the Middle Horizon (500-1000 CE) impact on local diet. American Journal of Physical Anthropology 148:62-72.

Isbell WH. 2008. Wari and Tiwanaku: international identities in the Central Andean Middle Horizon. In: Silverman H, Isbell WH, editors. The handbook of South American archaeology. p. 731-759. Springer: New York. 
Janusek JW. 2004. Identity and power in the ancient Andes: Tiwanaku cities through time. New York: Routledge.

Jennings J, Tung TA, Yépez Álvarez W, Quequezana Lucano GC, López Hurtado MA. 2015. Shifting local, regional, and interregional relations in Middle Horizon, Peru: evidence from La Real. Latin American Antiquity 26(3):382-400.

Kolata A. 1993. The Tiwanaku: portrait of an Andean civilization. Cambridge (MA): Basil Blackwell.

Le Paige G. 1963. Introducción. Congreso Internacional de Arqueología de San Pedro de Atacama/Anales de la Universidad del Norte 2:4.

Le Paige G. 1964. El precerámico en la cordillera atacameña y los cementerios del período agroalfarero de San Pedro de Atacama. Anales de la Universidad del Norte 3:5-275.

Le Paige G. 1972/1973. Tres cementerios indígenas en San Pedro de Atacama y Toconao. Actas del VI Congreso de Arqueología Chilena (Octubre de 1971). Boletín de Prehistoria, número especial.

Le Paige G. 1976. Nuevas fechas radiocarbónicas de la zona de San Pedro de Atacama. Estudios Atacameños 4:145.

Llagostera A. 1995. El componente Aguada en San Pedro de Atacama. Boletín del Museo Chileno de Arte Precolombino 6:9-34.

Llagostera A. 1996. San Pedro de Atacama: nodo de complementariedad reticular. Estudios y Debates Regionales Andinos 96:17-42.

Llagostera A. 2004. Los antiguos habitantes del Salar de Atacama: prehistoria atacameña. Santiago: Pehuen.

Llagostera A, Barón AM, Bravo L. 1984. Investigaciones Arqueológicas en Tulor 1. Estudios Atacameños 7:105-115.

Llagostera A, Costa Junqueira MA. 1999. Patrones de asentamiento en la época agroalfarera de San Pedro de Atacama (norte de Chile). Estudios Atacameños 17:175-206.

Llagostera A, Torres CM, Costa Junqueira MA. 1988. El complejo psicotrópico en Solcor-3 (San Pedro de Atacama). Estudios Atacameños 9:61-98.

Marsteller SJ, Torres-Rouff C, Knudson KJ. 2011. Pre-Columbian Andean sickness ideology and the social experience of leishmaniasis: a contextualized analysis of bioarchaeological and paleopathological data from San Pedro de Atacama, Chile. International Journal of Paleopathology 1(1):24-34.

McRostie V. 2014. Arboricultura y silvopastoralismo en el período Formativo $(1.400 \mathrm{aC}-500 \mathrm{dC})$ de la cuenca del Salar de Atacama. Chungara 46(4):543-557.

Muñoz I, Agüero C, Valenzuela D. 2016. Poblaciones prehispanicas de los Valles Occidentales del norte de Chile: desde el Periodo formativo al Intermedio Tardío (ca. 1.000 años a.C. a
1.400 años d.C.). In: Falabella F, Uribe M, Sanhueza L, Aldunate C, Hidalgo J, editors. Prehistoria en Chile: Desde sus Primeros Habitantes Hasta Los Incas. p. 181-238. Santiago de Chile: Editorial Universitaria.

Neves WA, Costa MA. 1998. Adult stature and standard of living in the prehistoric Atacama Desert. Current Anthropology 39:278-281.

Niemeyer H, editor. 1963. Congreso Internacional de Arqueología de San Pedro de Atacama/Anales de la Universidad del Norte 2.

Niemeyer H. 1989. El escenario geográfico. In: Hidalgo J, Schiappacasse V, Niemeyer $\mathrm{H}$, Aldunate C, Solimano I, editors. Culturas de Chile: Prehistoria Desde sus Orígenes Hasta los Albores de la Conquista. Santiago de Chile: Editorial Universitaria. p. 1-12

Núñez L. 1963. Problemas en torno a la tableta de rapé. Congreso Internacional de Arqueología de San Pedro de Atacama/Anales de la Universidad del Norte 2:146-168.

Núñez L. 1965. Recientes fechados radiocarbónicos del norte de Chile. Estudios Arqueológicos 1:107-109.

Núñez L. 1966. Recientes fechados radiocarbónicos del norte de Chile. Boletín de la Universidad de Chile [Santiago] 50:51-56.

Núñez L. 1976. Registro regional de fechas radiocarbónicas en el norte de Chile. Estudios Atacameños 4:74-123.

Núñez L. 1991. Cultura y conflicto en los oasis de San Pedro de Atacama. Santiago de Chile: Editorial Universitaria.

Núñez L. 2007. Vida y Cultura en el oasis de San Pedro de Atacama. Santiago de Chile: Editorial Universitaria.

Núñez L, Dillehay T. 1995. Movilidad giratoria, armonía social y desarrollo en los Andes Meridionales: patrones de tráfico e interacción económica. Antofagasta, Chile: Universidad del Norte.

Núñez L, Grosjean M, Cartajena I. 2005. Ocupaciones humanas y paleoambientes en la Puna de Atacama. San Pedro de Atacama: Universidad Católica del Norte.

Núñez L, Grosjean M, Cartajena I. 2010. Sequential analysis of human occupation patterns and resource use in the Atacama desert. Chungara 42(2):363-391.

Oakland A. 1992. Textiles and ethnicity: Tiwanaku in San Pedro de Atacama, North Chile. Latin American Antiquity 3:316-340.

Orellana M. 1962. Recientes investigaciones arqueológicas en San Pedro de Atacama. Centro de Estudios Antropológicos. Apartado de la Revista Mensaje XI(108).

Orellana M. 1963. Problemas de la arqueología de San Pedro de Atacama y sus alrededores. Congreso Internacional de Arqueología San Pedro de Atacama. Congreso Internacional de 
Arqueología de San Pedro de Atacama/Anales de la Universidad del Norte 2:29-45.

Orellana M. 1964. Acerca de la cronología del complejo cultural San Pedro de Atacama. Antropología, Universidad de Chile [Santiago], No. 2. p. 96-104.

Orellana M. 1985. Relaciones culturales entre Tiwanaku y San Pedro de Atacama. Diálogo Andino 4:247-258.

Pestle WJ, Torres-Rouff C, Hubbe M. 2016. Modeling Diet in Times of Change: The Case of Quitor, San Pedro de Atacama, Chile. Journal of Archaeological Science: Reports 7:82-93.

Pestle WJ, Torres-Rouff C, Hubbe M, Smith EK. 2017. Eating in or dining out: modeling diverse dietary strategies in Middle Period San Pedro de Atacama, Chile. Archaeological and Anthropological Sciences 9(7):1363-1377.

Salazar D, Niemeyer H, Horta H, Figueroa V, Manríquez H. 2014. Interaction, social identity, agency and change during Middle Horizon San Pedro de Atacama (northern Chile): a multidimensional and interdisciplinary perspective. Journal of Anthropological Archaeology 35: 135-152.

Serracino G. 1980. Tiwanaku desde San Pedro de Atacama. Estudios arqueologicos 5:95-106.

Stovel EM. 2001. Patrones funerarios de San Pedro de Atacama y el problema de la presencia de los contextos Tiwanaku. Boletín de Arqueología PUCP 5:375-395.

Stovel EM. 2005. The Archaeology of identity construction: ceramic evidence from Northern Chile. In: Funari P, Zarankin A, Stovel E, editors. Global archaeological theory: contextual voices and contemporary thoughts. New York: Springer/Kluwer. p. 145-166.

Stovel EM. 2013. Prehistoric Atacameño ceramic styles and chronology revisited. Chungara Revista de Antropología Chilena 45(3): 371-385.

Tarragó M. 1968. Secuencias culturales de la etapa agroalfarera de San Pedro de Atacama (Chile). Proceedings of the 37 th International Congress of Americanists, Mar del Plata, Buenos Aires, Argentina 2:119-145.

Tarragó M. 1989. Contribución al conocimiento arqueológico de las poblaciones de los oasis de San Pedro de Atacama en relación con los otros pueblos puneños, en especial, el sector septentrional del Valle Calchaquí [unpublished PhD dissertation]. Facultad de Humanidades y Artes, Universidad Nacional de Rosario, Rosario, Argentina.
Torres CM, Conklin WJ. 1995. Exploring the San Pedro de Atacama/Tiwanaku relationship. Worldwide Archaeology Series 13:78-108.

Torres CM, Repke DB, Chan K, McKenna D, Llagostera A, Schultes RE. 1991. Snuff powders from pre-Hispanic San Pedro de Atacama: Chemical and contextual analysis. Current Anthropology 32(5):640-649.

Torres-Rouff C. 2008. The influence of Tiwanaku on life in the Chilean Atacama: mortuary and bodily perspectives. American Anthropologist 110: 325-337.

Torres-Rouff C. 2011. Hiding inequality beneath prosperity: patterns of cranial injury in Middle Period San Pedro de Atacama, Northern Chile. American Journal of Physical Anthropology 146(1):28-37.

Torres-Rouff C, Costa Junqueira MA. 2006. Interpersonal violence in prehistoric San Pedro de Atacama, Chile: behavioral implications of environmental stress. American Journal of Physical Anthropology 130(1):60-70.

Torres-Rouff C, Hubbe M. 2013. The sequence of human occupation in the Atacama oases, Chile: a radiocarbon chronology based on human skeletal remains. Latin American Antiquity 42(3):330-344.

Torres-Rouff C, Hubbe M, Pestle WJ. 2018. Wearing the marks of violence: unusual trauma patterning at Coyo Oriental, northern Chile (AD 400-1000). American Journal of Physical Anthropology 167(1):32-45.

Uribe M. 2002. Sobre alfarería, cementerios, fases y procesos durante la prehistoria tardía del desierto de Atacama (800-1600 DC). Estudios Atacameños 22:7-31.

Uribe M, Agüero C. 2001. Alfarería, textiles y la integración del Norte Grande de Chile a Tiwanaku. Boletín de Arqueología PUCP 5:397-426.

Uribe M, Agüero C. 2004. Iconografía, alfarería y textilería Tiwanaku: elementos para una revisión del período Medio en el Norte Grande de Chile. Actas del XV Congreso Nacional de Arqueología Chilena. Chungara Revista de Antropología Chilena 36 Número Especial Tomo 2:1055-1068.

Uribe M, Santana-Sagredo F, Maturana A, Sergio Flores C, Agüero C. 2016. San Pedro de Atacama y la Cuestión Tiwanaku en el Norte de Chile: Impresiones a Partir de un Clásico Estudio Cerámico y la Evidencia Bioarqueológica Actual (400-1.000 D.C.). Chungara, Revista de Antropología Chilena 48(2):173-198. 